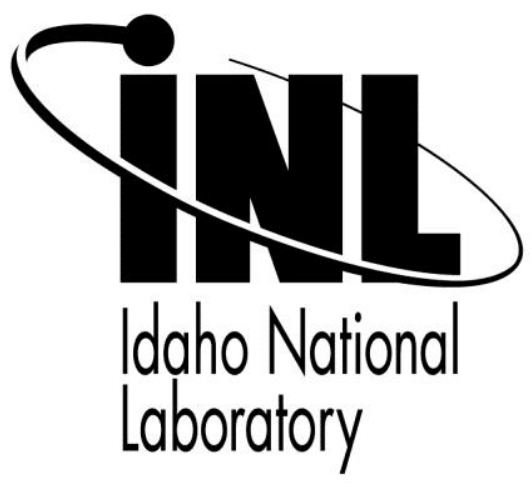

\title{
Extension of the
} Advanced Test Reactor Operating Envelope via Enhanced Reactor Physics Validation Techniques

\author{
D.W. Nigg, J.W. Nielsen, D.R. Norman \\ (Idaho National Laboratory)
}

\section{March 2017}

This is an accepted manuscript of a paper intended for publication in a journal. This document was prepared as an account of work sponsored by an agency of the United States Government. Neither the United States Government nor any agency thereof, or any of their employees, makes any warranty, expressed or implied, or assumes any legal liability or responsibility for any third party's use, or the results of such use, of any information, apparatus, product or process disclosed in this report, or represents that its use by such third party would not infringe privately owned rights. The views expressed in this paper are not necessarily those of the United States Government or the sponsoring agency.

Prepared for the U.S. Department of Energy Office of Nuclear Energy

Under DOE Idaho Operations Office Contract DE-AC07-05ID14517 

Extension of the Advanced Test Reactor Operating Envelope via Enhanced Reactor Physics Validation Techniques

Joseph W. Nielsen, David W. Nigg, and Daren R Norman

Idaho National Laboratory, Idaho Falls, ID USA

joseph.nielsen@inl.gov

dwn@inl.gov

daren.norman@inl.gov

Manuscript prepared for submittal to Nuclear Technology (Revision 2 - STIMS) 


\section{ABSTRACT \\ Extension of the Advanced Test Reactor Operating Envelope via Enhanced Reactor Physics Validation Techniques}

The Korea Atomic Energy Research Institute is currently in the process of qualifying a Low-Enriched Uranium fuel element design for the new Ki-Jang Research Reactor (KJRR). As part of this effort, a prototype KJRR fuel element was irradiated for several operating cycles in the Northeast Flux Trap of the Advanced Test Reactor (ATR) at the Idaho National Laboratory. The KJRR fuel element contained a very large quantity of fissile material $\left(618 \mathrm{~g}{ }^{235} \mathrm{U}\right)$ in comparison with historical ATR experiment standards $(<1 \mathrm{~g}$ ${ }^{235} \mathrm{U}$ ), and its presence in the ATR flux trap was expected to create a neutronic configuration that would be well outside of the approved validation envelope for the reactor physics analysis methods used to support ATR operations.

Accordingly it was necessary to conduct an extensive set of new low-power physics measurements in the ATR Critical Facility (ATRC), with the objective of expanding the validation envelope for the computational reactor physics tools used to support ATR operations and safety analysis to include the planned KJRR irradiation in the ATR and similar experiments that are anticipated in the future.

The computational and experimental results have demonstrated that the neutronic behavior of the KJRR fuel element in the ATRC is well-understood, both in terms of its general effects on ATRC core reactivity and fission power distributions, its effects on the calibration of the core lobe power measurement system, as well as in terms of its own internal fission rate distribution and total fission power per unit ATRC core power. Taken as a whole, these results have significantly extended the ATR physics validation envelope, thereby enabling an entire new class of irradiation experiments.

Key Words: Research Reactors, Experiments, Physics, Fuel Testing 


\subsection{INTRODUCTION}

The Korea Atomic Energy Research Institute (KAERI) is conducting the Ki-Jang Research Reactor (KJRR) project to construct a new dedicated radioisotope production facility in the Ki-Jang province of South Korea. They are currently in the process of qualifying a new fuel element design. As part of this effort, a prototype KJRR fuel element is being irradiated for several operating cycles in the Northeast Flux Trap (NEFT) of the Advanced Test Reactor (ATR) at the Idaho National Laboratory (INL). The KJRR fuel element introduces an unprecedentedly large quantity $\left(618 \mathrm{~g}{ }^{235} \mathrm{U}\right)$ of fissile material by historical ATR experiment standards ( $\sim \mathrm{g}$ or less) into a flux trap, and its presence was expected to creates a neutronic configuration that would be well outside of the validation envelope for the reactor physics analysis methods used to support ATR operations. Furthermore, the active height of the KJRR fuel element is $60 \mathrm{~cm}$, less than half of the height of the ATRC core fuel elements. This produces non-standard axial power distributions in the core fuel elements surrounding the NEFT that needed to be quantified. Accordingly it was necessary to conduct an extensive set of new physics measurements in the ATRC, with the objective of expanding the validation envelope to include the KJRR irradiation in the ATR and similar experiments that are anticipated in the future.

\subsection{FACILITY DESCRIPTION}

The ATR is one of only a few high-power research reactors (HPRR) of its type in the world, with a variety of missions involving accelerated testing of nuclear fuel and other materials in a very high neutron flux $\left(5.0 \times 10^{14} \mathrm{n} / \mathrm{cm}^{2}\right.$ s) environment, medical and industrial isotope production, and other applications. The ATR is a highly-

heterogeneous, light-water and beryllium moderated, beryllium reflected, light-water cooled system with a single row of highly-enriched $\left(93 \%{ }^{235} \mathrm{U}\right)$ plate-type fuel elements arranged in a closed serpentine pattern surrounding nine individual flux traps and other experiment positions as shown in Figure 1. The active core height is 4 feet $(1.22 \mathrm{~m})$. It has a companion critical facility, the ATR Critical Facility ATRC (Figure 2) that is basically an open-pool copy of the ATR that typically operates at approximately $600 \mathrm{~W}$ and produces thermal neutron flux levels in the flux traps in the range of $1.0 \times 10^{9} \mathrm{n} / \mathrm{cm}^{2}-\mathrm{s}$. The ATRC facility is typically used with prototype experiments to characterize in advance, with precision and accuracy, the expected changes in core reactivity and fission power distribution for the same experiments in the ATR. Useful physics data can also be obtained for evaluating the worth and calibration of control elements as well as thermal and fast neutron flux distributions.
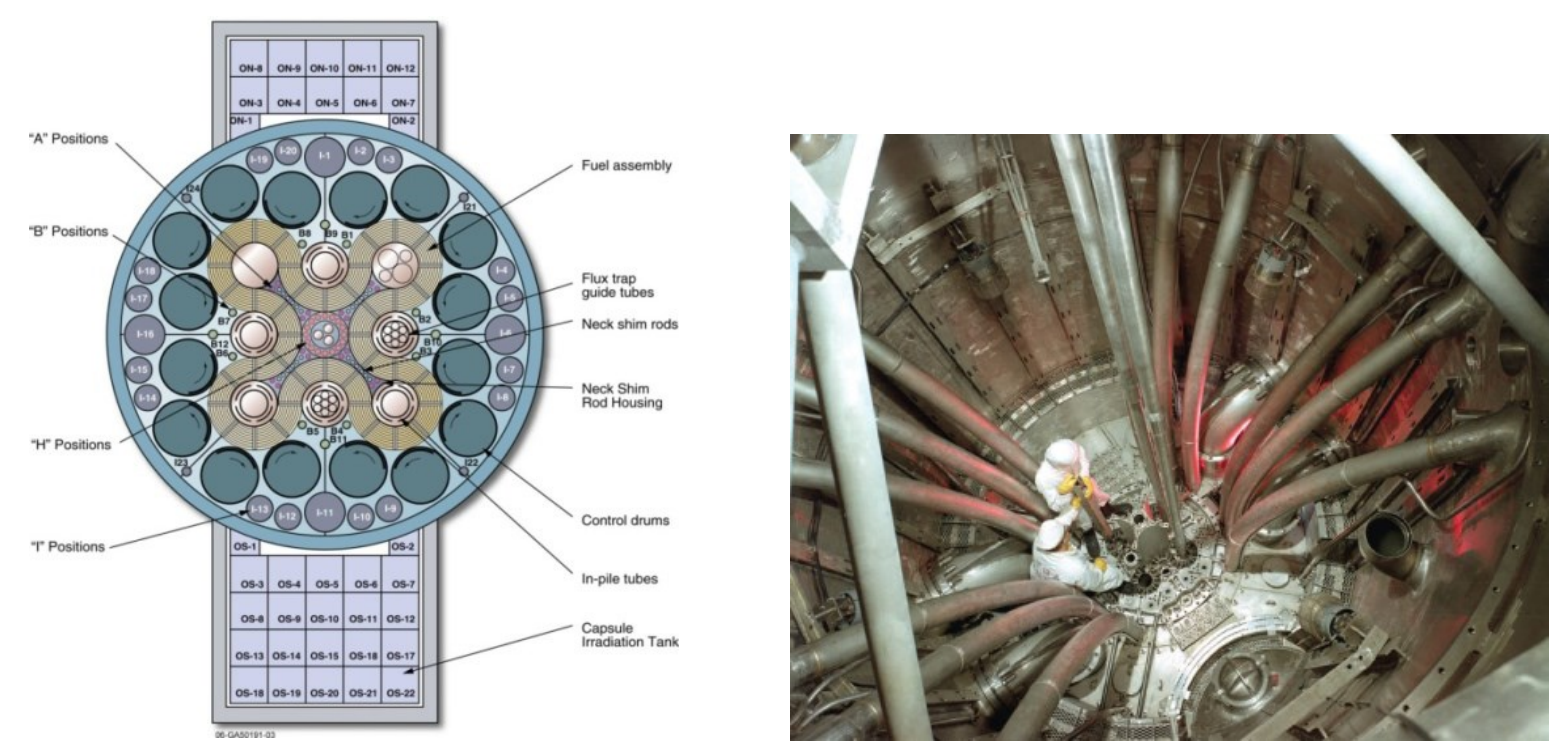

Figure 1. Core and reflector geometry of the Advanced Test Reactor in the transverse midplane (L) and view into the ATR pressure vessel during construction $(\mathrm{R})$ 

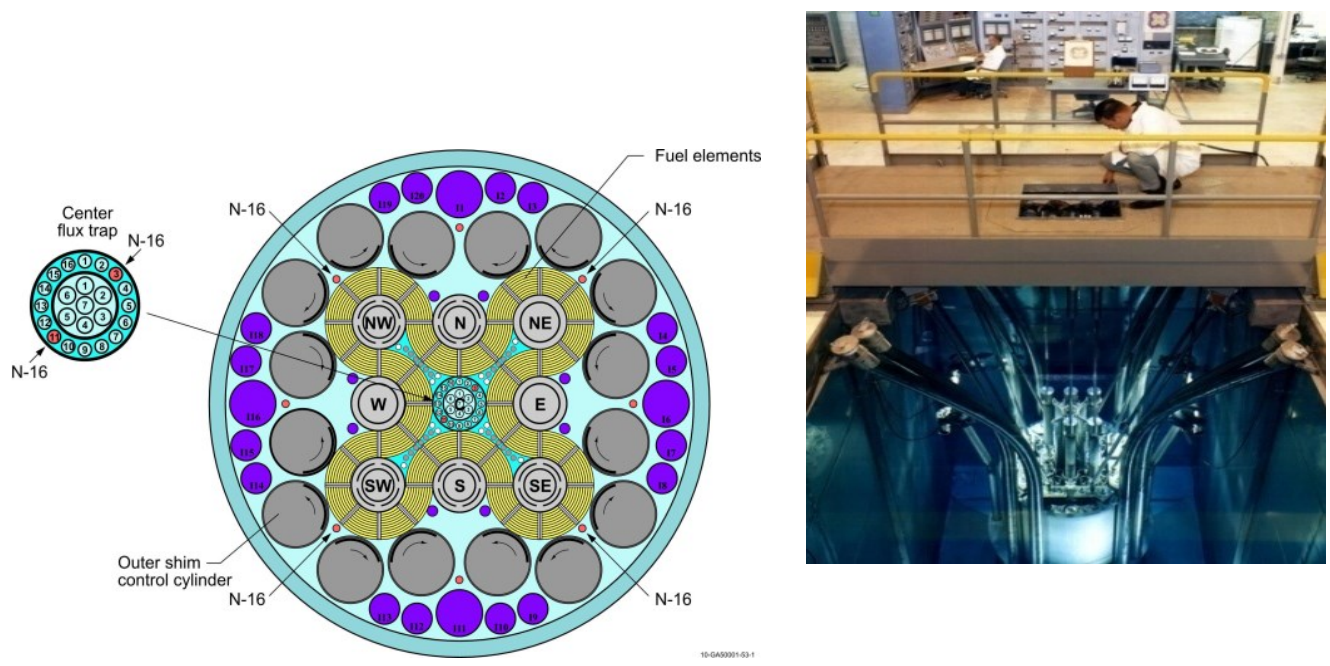

Figure 2. Transverse cross-section of the ATR Critical Facility (L) and view into the ATRC shielding tank (R).

\subsection{METHODS AND MATERIALS}

The INL recently completed a focused multiyear effort to upgrade legacy computational reactor physics software tools and protocols used for support of core fuel management, experiment management, and safety analysis for the ATR and its companion critical facility (ATRC) at the INL. This was accomplished through the introduction of modern high-fidelity computational software and protocols, with appropriate verification and validation (V\&V) according to applicable national standards. A suite of well-recognized stochastic and deterministic transport theory based reactor physics codes and their supporting nuclear data libraries (HELIOS [1], NEWT [2], ATTILA [3], KENO6 [4], and MCNP5 [5]) is in place at the INL for this purpose, and corresponding baseline models of the ATR and ATRC are operational. Furthermore, a capability for rigorous sensitivity analysis and uncertainty quantification based on the TSUNAMI [6] system has been implemented. Finally, we also have been incorporating the MC21 [7] and SERPENT [8] stochastic simulation and depletion codes into the new suite on a trial basis as additional tools for $\mathrm{V} \& \mathrm{~V}$ in the near term and possibly as advanced platforms for full 3-dimensional Monte Carlo based ATR fuel cycle analysis and fuel management in the longer term.

Figure 3 shows the suite of new tools, how they generally relate to one another, and how they are applied to the ATR. This illustration is not a computational flow chart or procedure per se. Specific computational protocols using the tools shown in Figure 3 for routine ATR support applications are promulgated in approved procedures and other operational documentation. The most recent release of the Evaluated Nuclear Data Files (ENDF/B Version 7) is generally used to provide the basic cross section data and other nuclear parameters required for all of the modeling codes. The ENDF physical nuclear data files are processed into computationally-useful formats using the NJOY or AMPX codes as applicable to a particular module [9], as shown at the top of Figure 3.

On the experimental side of the effort, several new benchmark-quality code validation measurements have been conducted using the ATR and the ATRC. Results for the first six experiments, focused on detailed neutron spectrum measurements within the Northwest Large In-Pile Tube (NW LIPT), along with measurements of neutron flux spectra within the core fuel elements surrounding the NW LIPT and the diametrically opposite Southeast IPT and within the Southeast IPT have been reported $[9,10,11]$. More recently we completed a series of experiments in the ATRC focused on computation and validation of the fuel element-to-element power distribution [12]. In the work summarized here we present computational and experimental results for an additional set of new ATRC experiments designed specifically to extend the ATR physics code validation envelope to a new class of irradiations involving placement of unprecedentedly large quantities of fissile material in the main flux traps. These new types of irradiations not only involve much more fuel - they also feature geometric experiment configurations that introduce significant flux and power distribution perturbations in the transverse and axial dimensions of the core to a degree that heretofore had not been encountered. 


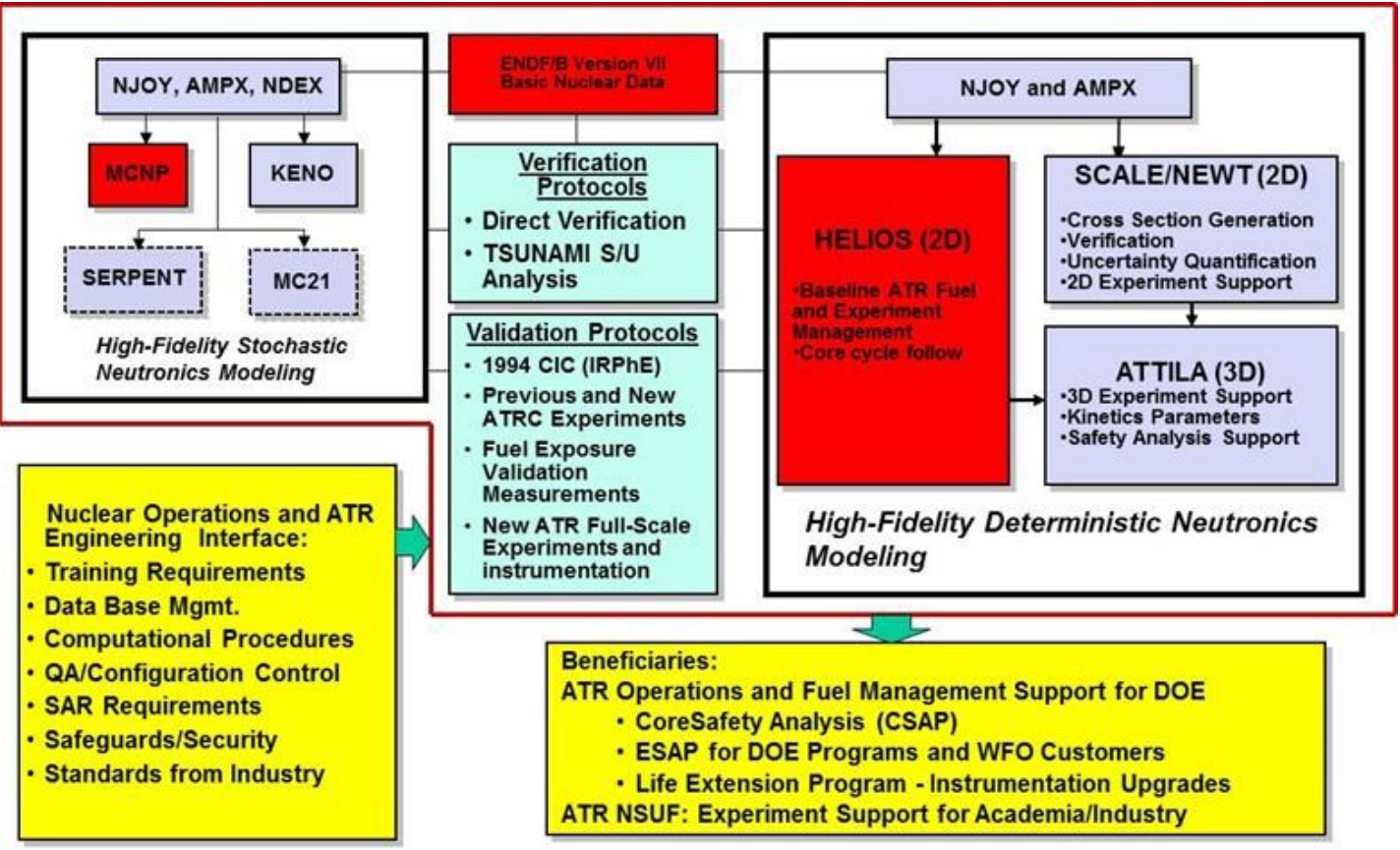

Figure 3. Advanced computational tool suite for the ATR and ATRC, with supporting verification, validation and administrative infrastructure.

\subsection{Experiment Description}

The KJRR fuel element contains a very large quantity of fissile material $\left(618 \mathrm{~g}{ }^{235} \mathrm{U}\right)$ in comparison with historical ATR experiment standards. Its presence in the ATR flux trap was expected to create a neutronic configuration that would be well outside of the approved validation envelope for the reactor physics analysis methods used to support ATR operations. Accordingly it was necessary to conduct an extensive set of new low-power physics measurements in the ATRC, with the objective of expanding the validation envelope for the computational reactor physics tools used to support ATR operations and safety analysis to include the planned KJRR irradiation in the ATR and similar experiments that are anticipated in the future.

The KJRR will employ a plate-type driver fuel assembly. The fuel meat consists of uranium with $7 \mathrm{wt} \%$ molybdenum (U-7Mo) metallic alloy particles dispersed in a blended matrix of pure aluminum and $5 \mathrm{wt} \%$ silicon. The silicon was not included in the models and will have a negligible effect on neutronic interactions. The uranium enrichment will be $19.75 \mathrm{wt} \%{ }^{235} \mathrm{U}$. Aluminum alloy 6061(Al-6061) will be used as the fuel plate cladding material (Figure 3). The active height of the KJRR fuel plates is $60 \mathrm{~cm}$, less than half of the height of the ATRC core fuel elements as noted previously.

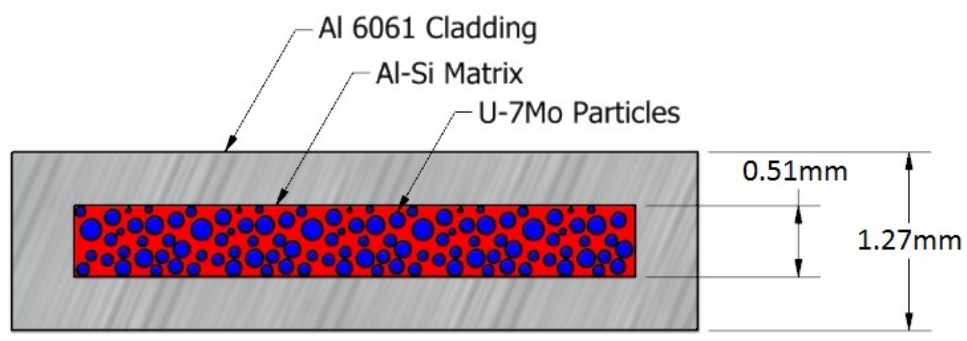

Figure 3. KJRR Fuel Plate Cross Section 


\section{[Type here]}

Two fuel meat uranium densities of 8.0 and $6.5 \mathrm{~g}-\mathrm{U} / \mathrm{cm}^{3}$ are used for the 19 interior plates and the 2 exterior plates of the KJRR fuel assembly, respectively. Fuel plate fabrication is accomplished by hot roll bonding processes. Each of the 21 flat fuel plates is incorporated into a fuel assembly by swaging in the grooves of Al-6061 side plates resulting in a fissile loading of $618 \mathrm{~g}{ }^{235} \mathrm{U}$ for each KJRR fuel assembly (Figure 4).

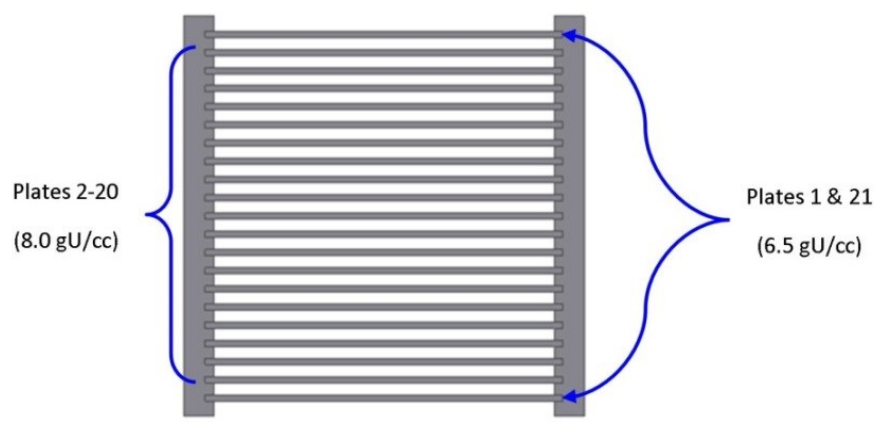

Figure 4. KJRR Fuel Assembly, Top View

\subsection{Computational Model Description}

MCNP5 [5] was used to model and evaluate the KJRR experiment in the ATRC and the ATR. The validated MCNP5 model can then be used as a tool for cross-validating the other transport codes in the suite described previously. A diagram of the MCNP5 ATRC model is shown in Figure 5. Each of the fuel plates was modeled using nominal dimensions. The KJRR fuel element was modeled in MCNP5 with nominal U-235 fuel loading and nominal conditions. The nominal U-235 loading is $618.30 \mathrm{~g}$ with an enrichment of $19.75 \% \mathrm{U}-235$ for a total U loading of $3130.6 \mathrm{~g}$. In order to achieve the desired neutronic performance of the core for the KJRR experiment campaign, several ATRC experiment positions were reconfigured and various different types of ATR/ATRC core fuel elements (relative to the standard fuel elements normally used) were loaded. These changes were reflected in the model as well.

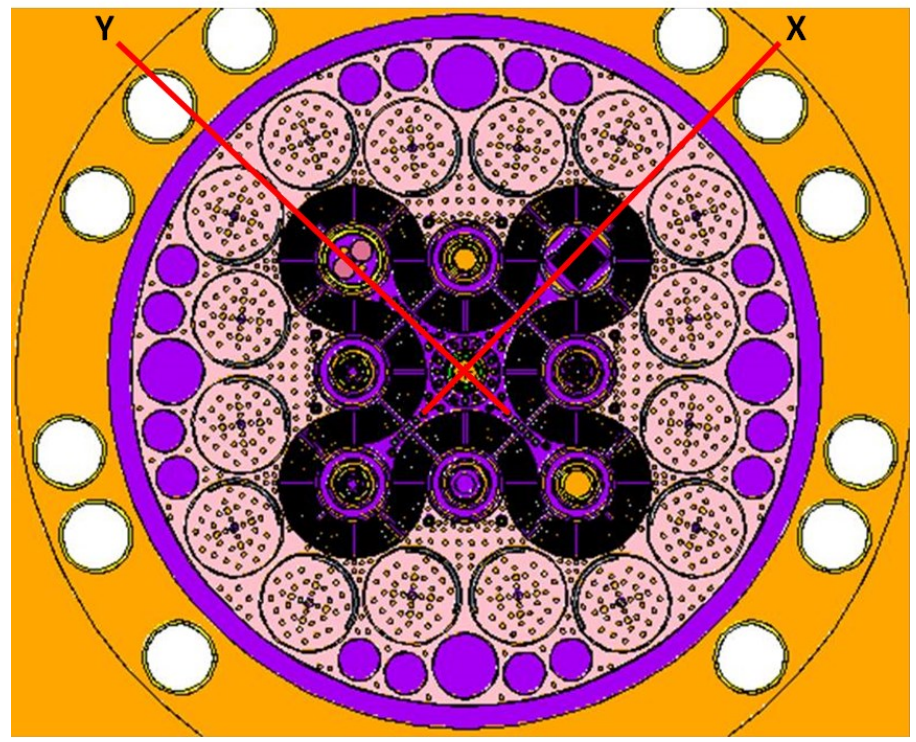

Figure 5. Transverse section of ATRC MCNP core model at the axial midplane showing positioning of the KJRR experiment in the Northwest Large In-Pile Tube and defining the X-Y coordinate system used for fission wire positioning in the KJRR fuel element. The $\mathrm{Z}$-axis is perpendicular to the plane of the illustration, with $\mathrm{Z}=0$ at the axial midplane of the active ATRC core. 


\subsection{Measurement Description}

The measurements consisted of several critical shim position determinations at various hold points during the ATRC core reconfiguration and KJRR fuel element experiment insertion procedure [13] along with two detailed threedimensional neutron flux and power distribution measurements, with and without the KJRR fuel element installed. These latter two experiments, documented here, were designed to provide validation data for the fuel element-toelement ATRC core power distribution and for the 3D/2D reactivity and power distribution correction protocols used with the HELIOS-based ATR fuel cycle analysis methodology. In addition the measurements provided validation data for the KJRR fuel element total fission power and for the intra-element fission power distribution in the axial and transverse dimensions within the KJRR element. The measurements were conducted with the core and (in the second case) the KJRR experiment hardware heavily instrumented with standard U-Al fission wires [14] to measure fission rates at various locations of interest. The fission wires were composed of aluminum alloyed with $10 \%$ by weight fully-enriched $\left(93 \%{ }^{235} \mathrm{U}\right)$ uranium metal. All fission wire and flux wire measurements were assumed to have the historically demonstrated experimental uncertainty $(1 \sigma=5 \%)$

Figure 5 shows the coordinate system defined for specifying the location of fission wires in the KJRR experiment fuel element and Figure 6 shows the pattern for loading of fission wires into core fuel elements using Implex ${ }^{\mathrm{TM}}$ plastic "wands" to measure the intra-element fission distribution as well as the total element power [15]. Finally, measurements were conducted to obtain safety-related data on the distortion of the axial and transverse power distribution within the ATR core fuel elements surrounding the NE lobe, along with data to help quantify the effect of the KJRR experiment hardware on the calibration of the online lobe power measurement system in the ATR

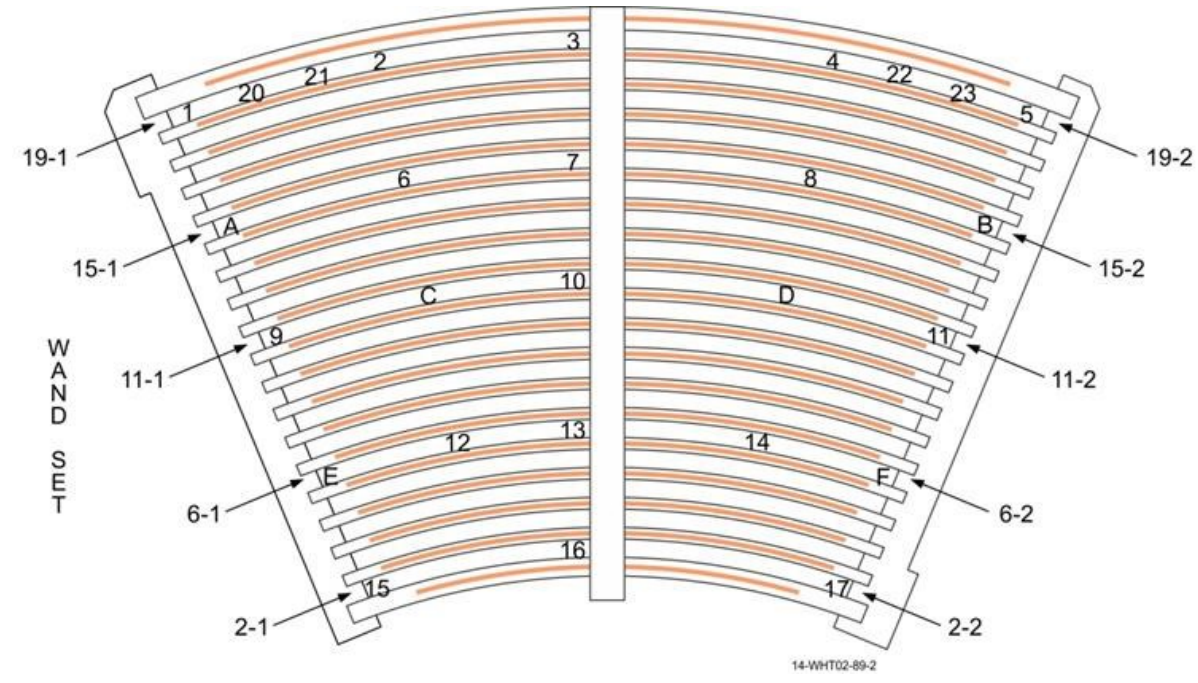

Figure 6. ATRC fuel element showing coolant channel numbering system and core fission wire positions at the transverse axial midplane. Standard wire positions for Irradiations are denoted by numbers 1-17. Wires can also be placed along the element vertical axis at intervals of $5.08 \mathrm{~cm}(2 ")$.

Figure 7 shows the fission wire location numbering system for the KJRR fuel element, referenced to the global coordinate system shown in Figure 5. The wires were positioned in the instrumented channels using special wands fabricated for the purpose (Figure 8). These wands were designed to hold a row of ten fission wires spaced in the channel at the axial midplane of the element. They also held paired wires, one wire on each side of the central rib, at 5 vertical positions separated by $5.08 \mathrm{~cm}(2 ")$ above the axial midplane and at 5 corresponding equally-spaced positions below the axial midplane. Fission wire wands were placed in Channels A,B,D,F,H,J,K,M,O,Q,S, and T. Each of the 12 wands had 30 wire positions, for a total of 360 wires that experimentally sample the fission rate at a representative set of locations in two perpendicular planes ( $X-Y$ plane at $Z=0$ and $X-Z$ plane at $Y=0$ ) passing through the KJRR fuel element. 


\section{[Type here]}

In the case of the core fission measurements, fission wires were placed in the 17 standard positions of the oddnumbered core fuel elements at the axial midplane (see Figure 6). In addition, Element 5 (see Figure 9) was instrumented with sets of axially-distributed wires in channels 2,6,11,15 and 19 along the central rib (Positions $16,13,10,7$ and 3). At each of these radial positions there were 12 wires above the axial midplane spaced on $5.08 \mathrm{~cm}$ (2") intervals starting $5.08 \mathrm{~cm}(2 ")$ above the midplane and 12 wires arranged correspondingly below the axial midplane.

The MCNP5 model results for the fission power distributions of interest were compared to the ATRC measurements using various statistical consistency assessment methodologies. By default in MCNP5, computed tally data are normalized to one fission source neutron produced in the system being modeled. Therefore, appropriate renormalization factors are used to convert tally results to absolute neutron fluxes, reaction rates, and heating rates etc. at the indicated reactor power.

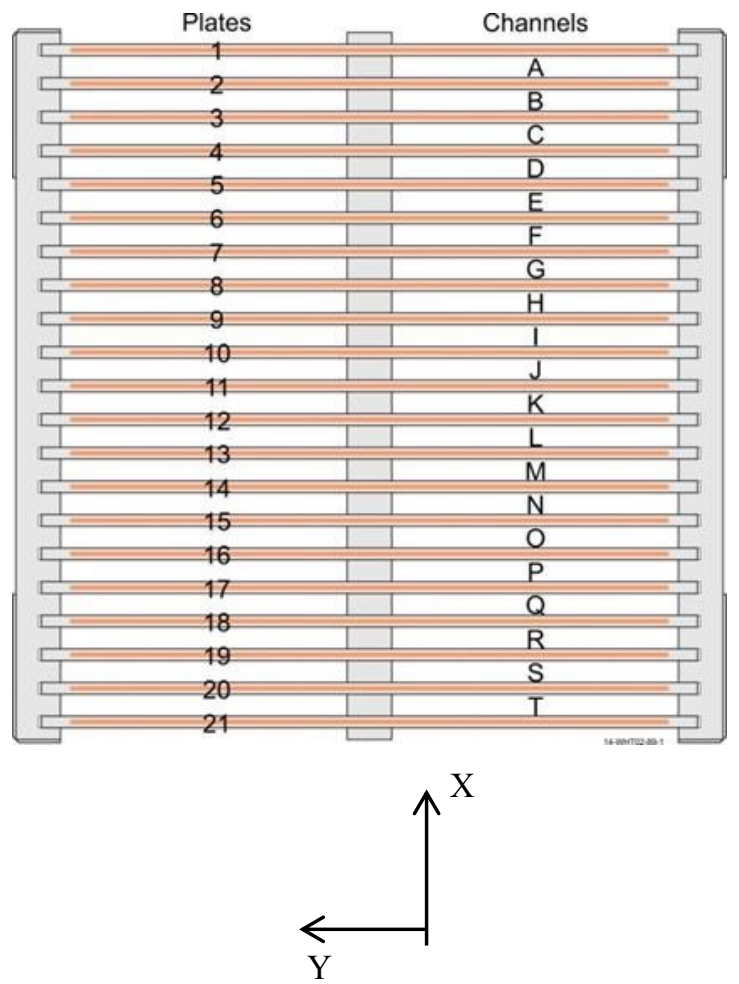

Figure 7. KJRR Fuel Element Plate and Channel Nomenclature. KJRR Plate 1 faces outward from the reactor center and KJRR Plate 21 faces the ATRC core center. Fission wire wands were placed in Channels

$\mathrm{A}, \mathrm{B}, \mathrm{D}, \mathrm{F}, \mathrm{H}, \mathrm{J}, \mathrm{K}, \mathrm{M}, \mathrm{O}, \mathrm{Q}, \mathrm{S}$, and $\mathrm{T}$ for the Irradiations. X-Position coordinates for fission wires are numbered from 120 , beginning in Channel $\mathrm{T}$ and going up (away from the core center). Y-Position coordinates for fission wires are numbered from 5 to -5 , beginning on the left.

Fission rates in the wires were measured by the INL Radiation Measurements Laboratory (RML) using standard procedures [14] based on gross beta counting. The results were reported in units of watts of fission energy released per gram of ${ }^{235} \mathrm{U}$ in the wire. The corresponding calculated fission rates were computed using $\mathrm{F} 7$ fission energy tallies available in the MCNP5 code. The MCNP5 fission energy deposition tally has units of MeV/g of ${ }^{235} \mathrm{U}$ in the region of interest per fission source neutron generated in the entire model geometry as noted above. Thus the tallies must be renormalized to the total number of fission source neutrons generated per unit time in the reactor at the operating power used for the experiment.

The purpose of this analysis is to document the validation of the MCNP5 ATRC model for the KJRR experiment. The experiments that were performed in the ATRC provide the most representative experimental conditions available without actually measuring in the ATR. The validation measurements and corresponding analyses provide bias and uncertainty parameters that can be extrapolated to modeling of ATR cycles containing the KJRR experiment hardware. For the purpose of this analysis, we are focused on the total power and internal power 
distribution within the KJRR fuel element and on the neutronic effects of the KJRR hardware on the surrounding ATR driver fuel. As such, the methodology applies to fission wires inserted in both the KJRR fuel element and the ATRC driver elements.

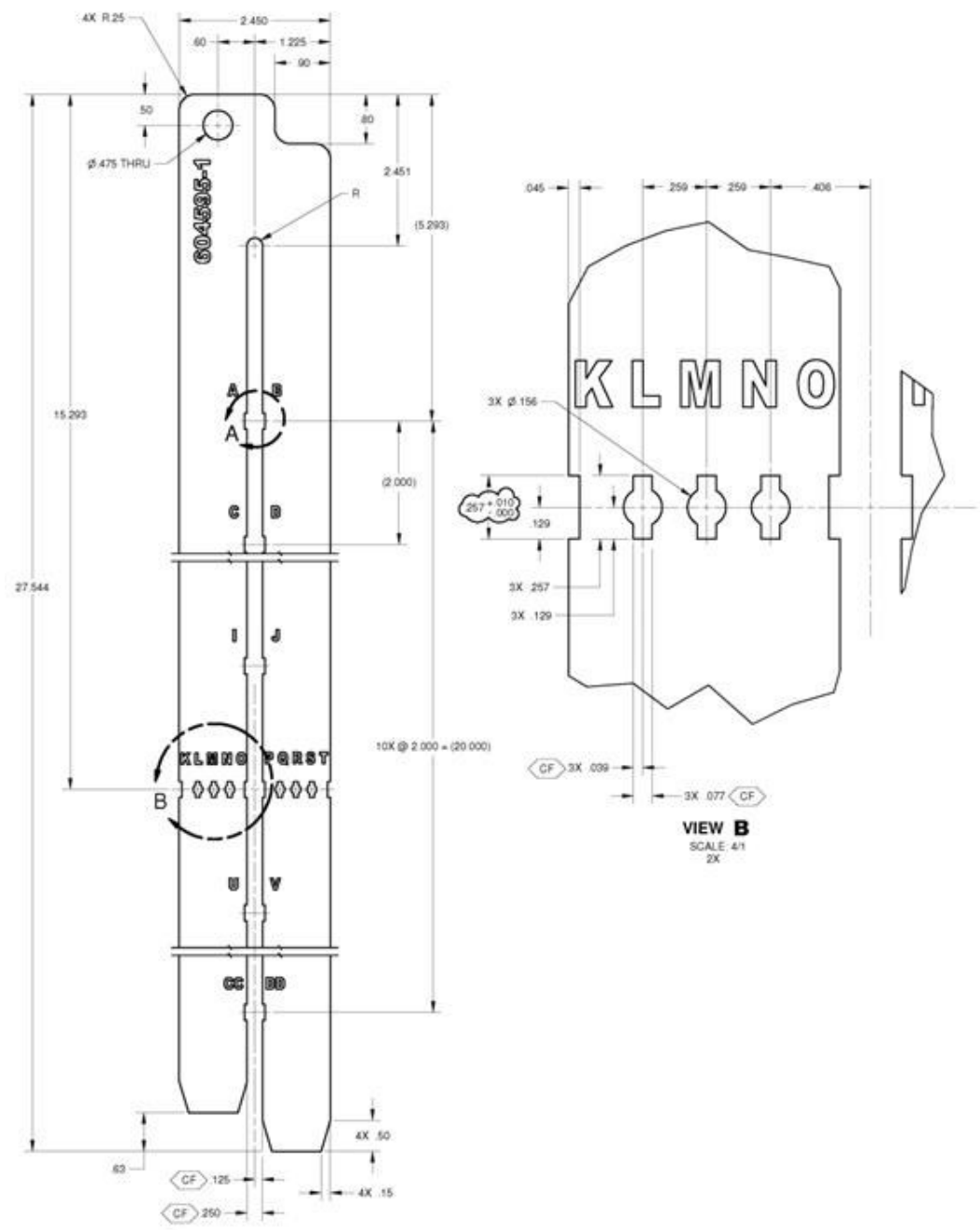

Figure 8. KJRR experiment fission wire wand geometry.

\subsection{ATRC CORE FUEL ELEMENT-TO-ELEMENT POWER DISTRIBUTIONS}

Fission power distributions for two ATRC configurations, referred to here as Irradiation 15-1 and Irradiation 15-2, were modeled with MCNP5 to provide comparisons between the ATRC core fission power distributions without and with the KJRR fuel element and its inner positioning basket installed, respectively. The Irradiation 15-1 models included a 3D model and an extruded 2D model, where the geometry and composition descriptions at the core midplane are assumed to be fully extruded in the axial direction. The calculated $\mathrm{K}_{\mathrm{eff}}$ values for the Irradiation $15-13 \mathrm{D}$ and $2 \mathrm{D}$ models were 0.98612 and 1.01835 , respectively. The calculated 3D/2D reactivity bias for this model is thus $3.2 \%$, consistent with previous ATR and ATRC experience. The measured fuel element powers for Irradiation 15-1, with the rotating shims set at 60.15 degrees are presented in Figure 9.

Figure 10 shows the results of a least-squares adjustment of the computed fuel element powers against the measured powers for the odd-numbered fuel elements (Figure 9) for the full 3-dimensional MCNP5 model of Irradiation 15-1. The adjustment protocol is described in detail in Reference [12]. The a priori element power uncertainties were 


\section{[Type here]}

conservatively assumed to be $10 \%$. Measurement uncertainty is $5 \%$ [15]. Critical shim rotation was $60.15^{\circ}$

(balanced). The maximum upward adjustment was about $4 \%$. The maximum downward adjustment was about $6 \%$. $68 \%$ of the adjustments fell within $\pm 2.9 \%(1 \sigma)$. The results of the adjustment are very consistent with historical experience for both ATR and ATRC, demonstrating a level of statistical consistency between MCNP5 3D model results and the corresponding experimental data that is well within the documented uncertainty (5\%) of the measurements themselves.

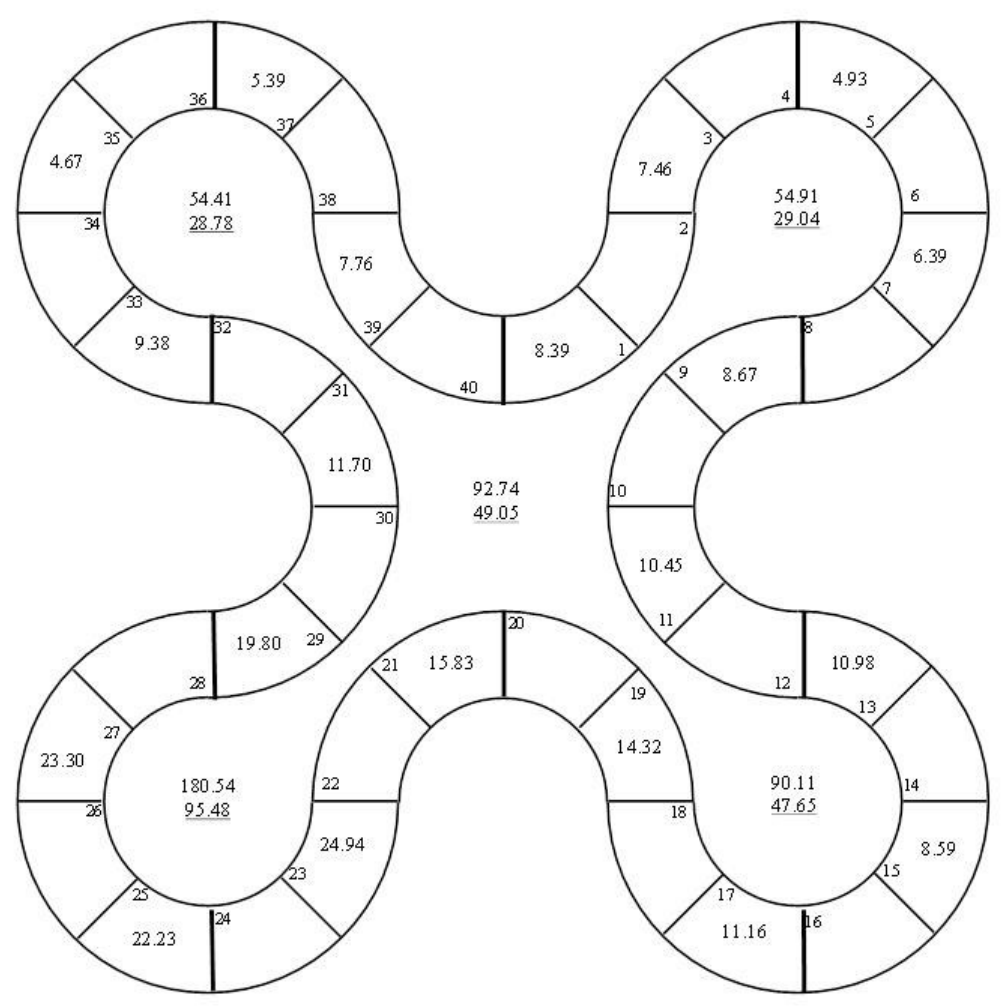

Figure 9. Measured core fuel element powers for ATRC Irradiation 15-1. Fuel Element Powers are shown in watts. Lobe Powers are shown in their respective lobes. Underlined power values are normalized to 250 watts. Total core power was reported to be 472.71 watts. Critical shim rotation was $60.15^{\circ}$.

Figure 11 shows the adjustment results where the a priori computed power distribution is taken from the extruded 2dimensional MCNP5 model of the 15-1 configuration. In this context "extruded" means that the 2-D model was constructed using a transverse geometry and composition description as found in an imaginary transverse plane corresponding to the axial midplane of the ATRC in the MCNP5 3D model. The 2D model has an imaginary axial height of $1 \mathrm{~cm}$ and specular reflection boundary conditions are imposed on the top and bottom surfaces. This situation corresponds to the $2 \mathrm{D}$ assumptions inherent in the equations solved by the HELIOS code used for ATR fuel cycle modeling. The maximum upward adjustment was about $5 \%$. The maximum downward adjustment was about $6 \% .68 \%$ of the adjustments fell within $\pm 2.6 \%(1 \sigma)$. Thus the adjustment procedure once again reveals excellent statistical consistency between the 2D computational model and the experimental results for Irradiation 151. Furthermore, the 2D-3D reactivity bias of $3.2 \%$ is also consistent with experience, as noted earlier. 


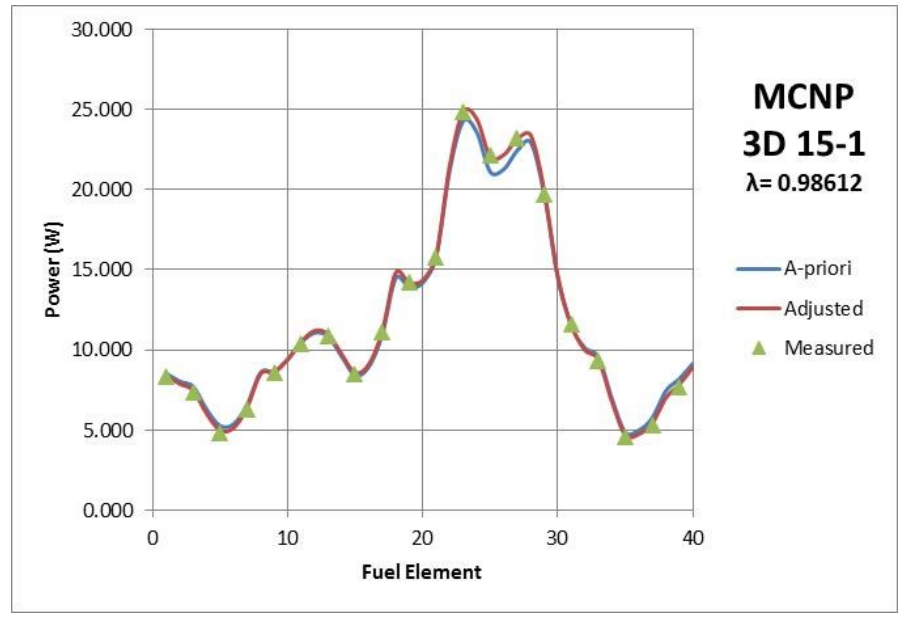

Figure 10. Fuel element fission rate distribution for KJRR Irradiation 15-1 (3D MCNP5 a priori).

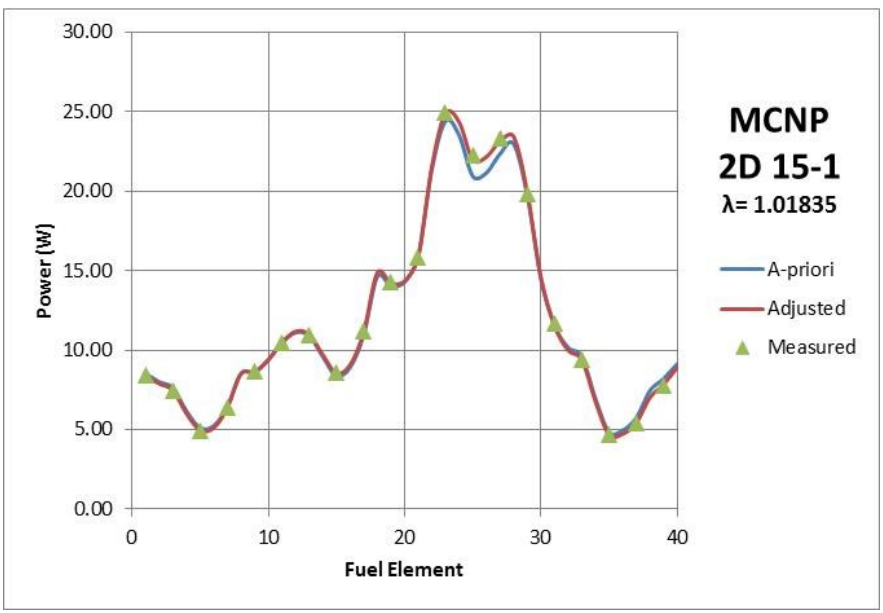

Figure 11. Fuel element fission rate distribution for KJRR Irradiation 15-1 (2D Extruded MCNP5 a priori).

The second detailed fission power distribution measurement in the ATRC (Irradiation 15-2) was conducted with the KJRR fuel element and its inner positioning basket placed in the NE Flux Trap. This experiment was designed to obtain detailed radial and axial measured fission power distributions in ATRC Core Fuel Element 5 (which was of the same type as is used in the ATR instead of a standard ATRC fuel element) and within the KJRR fuel element itself. Fission wires were placed in these two fuel elements as described earlier. The other odd-numbered core fuel elements were instrumented with fission wires in the same manner as in the Irradiation 15-1 configuration. Finally, the core fuel loading for Irradiation 15-2 also included four so-called "No-Boron" (burnable poison) fuel elements in the southwest lobe instead of standard ATRC elements in order to obtain the desired SW lobe power relative to the $\mathrm{NE}$ lobe power. The 15-2 configuration was evaluated using both 3D and corresponding 2D models to determine the appropriate corrections needed to account for axial effects caused by the presence of the KJRR fuel element. As noted earlier, the active axial height of the highly-reactive KJRR element is only $60 \mathrm{~cm}$ compared to $121.92 \mathrm{~cm}$ (48") in the case of the ATRC core fuel elements, so significant perturbations of the neutron flux and power distributions within the surrounding NE lobe core fuel elements were anticipated.

Figure 12 shows the measured core fuel element powers for the Irradiation 15-2 configuration. Note in this case however that the standard algorithm [15] used for computation of the total element powers in Figure 12 assumes that the axial peak-to-average fission power ratio in each element is 1.42 . As will be shown later, the axial power 


\section{[Type here]}

peaking factor in the elements surrounding the KJRR fuel element in the northeast lobe is approximately 1.63 with the KJRR fuel element in place, based on measurements in Element 5. If it is assumed that this peaking factor is the same for Elements 3-8 and that an average factor of 1.525 (average of 1.42. and 1.63) applies to Elements 2 and 9 in the same lobe, then the measured powers shown in Figure 13 must be corrected. This is because the midplane fission wires in the core fuel elements, whose fission powers are used to estimate the element power, are located in a higher peak than assumed in the standard measurement analysis protocol. The corrected measured element powers thus are 22.09, 18.63, 21.21, and 22.79 watts for Elements 3, 5, 7, and 9, respectively. The corresponding corrected NE lobe measured power is 169.47 watts and the corrected total reactor power is 724.63 watts.

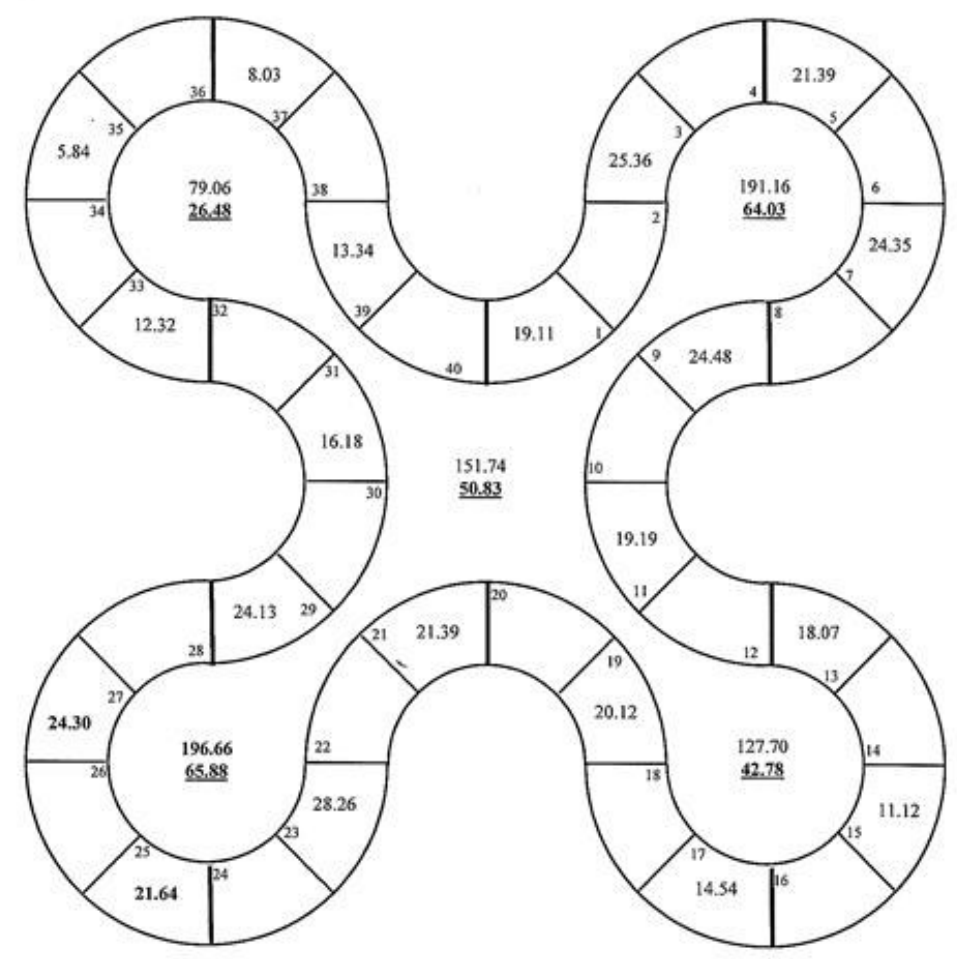

Figure 12. Measured core fuel element powers for ATRC Irradiation 15-2. Fuel element powers are shown in watts for the odd-numbered positions. Lobe Powers are shown in their respective lobes. The underlined power values are normalized to 250 watts. Total Power $=724.63$ Watts.

Figure 13 shows the results of the adjustment of the 3D computed core fuel element power distribution for the Irradiation 15-2 configuration against the corresponding measured powers, with the NE lobe measurements corrected for axial power distribution distortion effects as described above. The results show that the 3D model produces results that are reasonably consistent with the measurements. The maximum upward adjustment was about $7 \%$. The maximum downward adjustment was about $10 \%$. $68 \%$ of the adjustments fell within $\pm 5.5 \%(1 \sigma)$. However, in contrast to the case for Irradiation 15-1 there is a more discernable lobe-to-lobe tilt in the $a$ priori calculations relative to the measurements, with the computed NE and NW lobe powers about $7 \%$ higher than measured and the SE and SW lobe powers about 5\% lower than the corresponding measurements. This may be an indicator that the fissile content of the KJRR fuel element is slightly lower than reported in the design specifications, or that there is some other significant difference between the KJRR fuel element as modeled and the actual asfabricated element. Additionally, it is assumed that the RML algorithm used for computing core fuel element powers from the fission wire data is equally applicable to the various different types of fuel elements that were used in the core loading for Irradiation 15-2, which, strictly-speaking, may not be the case. 


\section{[Type here]}

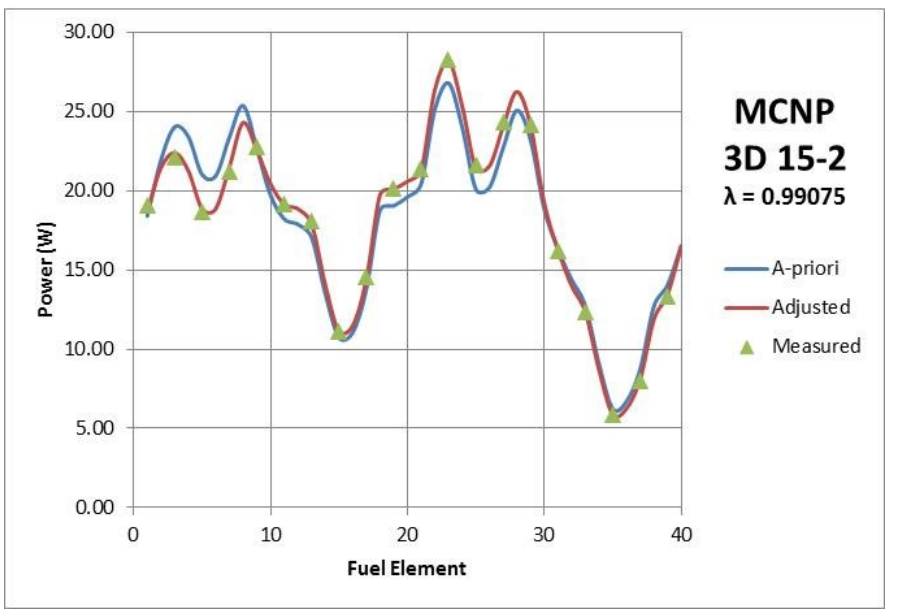

Figure 13. Fuel element fission rate distribution for KJRR Irradiation 15-2 (3D MCNP5 a priori). Critical shim rotation was $45.8^{\circ}$ (balanced).

Figure 14 shows the adjustment of the extruded 2D results for Irradiation 15-2. It can be seen that in the 2D case the a priori power distribution was not satisfactorily consistent with the measurements. Large adjustments, well beyond the uncertainty of the measurements, were needed to achieve consistency in a least-squares sense. The maximum upward adjustment was about $18 \%$. The maximum downward adjustment was about $22 \%$. $68 \%$ of the adjustments fell within $\pm 11.8 \%(1 \sigma)$. This poor result can be largely attributed to the fact that extrusion is not a good approximation for the 2D model in the case of the configuration for Irradiation 15-2, where an unusually large amount of fissile material (the KJRR fuel element) is confined to an active axial height that is only about half of that of the core fuel elements.

In order to more accurately account for axial effects in the 2D model of the configuration for Irradiation 15-2 it was necessary to reduce the ${ }^{235} \mathrm{U}$ and ${ }^{238} \mathrm{U}$ atom densities in the KJRR fuel element in the 2D model until its computed fraction of the total fission power in the 2D model (0.0311) closely matched that of the 3D model. This produced a reasonable $3 \mathrm{D} / 2 \mathrm{D}$ excess reactivity bias (3.1\%, consistent with the case for Irradiation 15-1) and brought the $a$ priori core fuel element powers into much better statistical consistency with the corresponding measurements, as can be seen in Figure 15. The maximum upward adjustment was about $6 \%$. The maximum downward adjustment was about $9 \%$. Furthermore, $68 \%$ of the adjustments fell within $\pm 5.0 \%(1 \sigma)$. As noted earlier, standard $3 \mathrm{D} / 2 \mathrm{D}$ correction modeling for the ATR would assume a fully extruded experiment over the $121.9 \mathrm{~cm}$ (48") axial length but this is not valid in the case of the $60 \mathrm{~cm}$ active length KJRR experiment. Thus a reduction in fissionable material density was required to produce a self-consistent $2 \mathrm{D}$ model that simultaneously reproduces the core excess reactivity and the element-to-element fission power distribution with acceptable accuracy.

It is interesting to note that the reduction in fissionable material density in the KJRR fuel element that produced the correct KJRR element power faction was on the order of $63 \%$, i.e. the physical uranium density in the element was multiplied by 0.37 . A simple axial homogenization of the KJRR fuel density over the axial height of the core would require a multiplier of 0.492 . However some additional reduction beyond simple homogenization was required in order to produce the correct KJRR element power fraction. For the most part this can probably be attributed to the fact that a shorter fuel element will have greater axial neutron leakage per unit power, and this must be accounted for when homogenizing the element over a larger axial dimension than its actual height. 


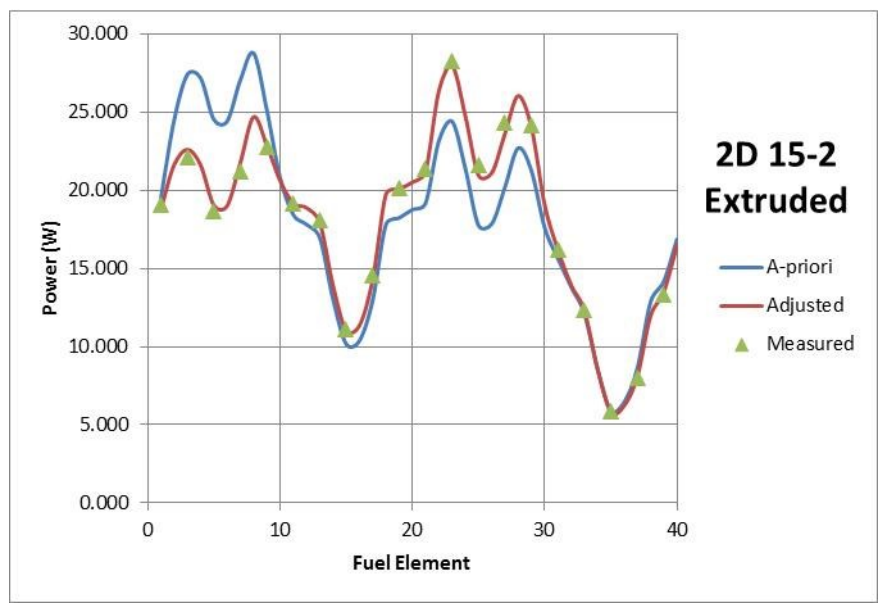

Figure 14. Fuel element fission rate distribution for KJRR Irradiation 15-2 (2D Extruded MCNP5 a priori)

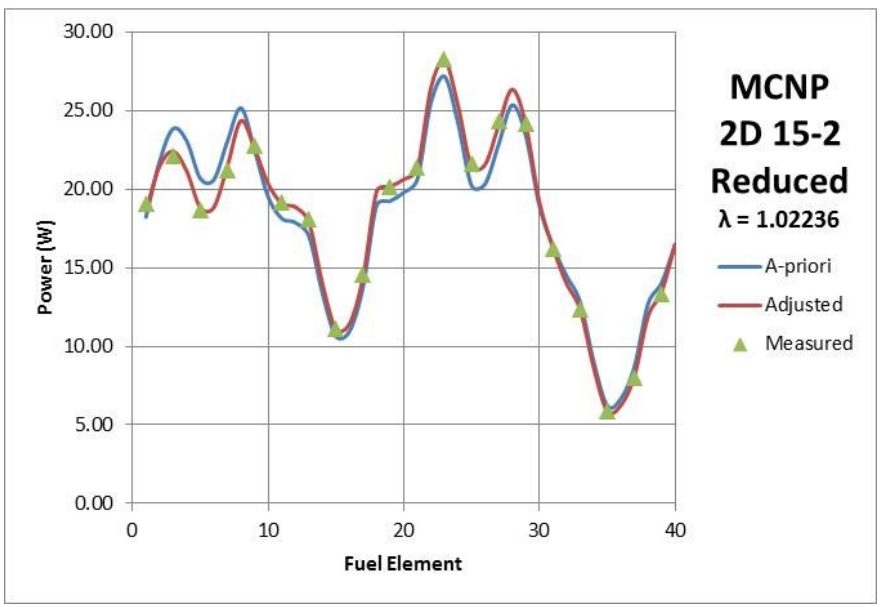

Figure 15. Fuel element fission rate distribution for KJRR Irradiation 15-2 (2D MCNP5 with reduced fuel density $a$ priori.)

\subsection{KJRR FUEL ELEMENT INTERNAL POWER DISTRIBUTION AND TOTAL FISSION POWER}

As a practical matter, fission rates in the KJRR fuel plates themselves at various locations could not be measured directly in the ATRC validation experiments, since such measurements would require destructive analyses that would render the element unusable for the actual long-term in the ATR. Accordingly, the KJRR fuel element was instrumented with 360 fission wires located throughout its active region. These wires were in close proximity to the actual KJRR fuel plates and they provided an indirect measure of the relative fission rate in the immediately adjacent region of the fuel plates between which each wire is located. The results of the wire measurements can be compared to corresponding MCNP5 calculations of the fission powers in the wires to assess the accuracy of the relative power distribution calculations produced by the computational model and to estimate the bias (if any) and the uncertainty of the computed total KJRR element power.

Comparisons of the calculated and measured relative fission power distributions for the wires and the corresponding implied total KJRR element power are presented in this section. Figure 16 shows, in a qualitative sense, the calculated and measured transverse two-dimensional relative fission rate distributions at the fission wire locations in the $\mathrm{X}-\mathrm{Y}$ axial midplane $(\mathrm{Z}=0)$ of the KJRR fuel element. As can be seen, the fuel element is heavily self-shielded, with the relative fission rates near the outer surfaces being roughly twice as large as the rates in the center. This is to be expected since a large fraction of the fissions occurring within the element are caused by neutrons entering the NE Flux Trap from the ATRC core, and these neutrons are attenuated as they pass through the various regions within the NE flux trap, especially the fueled regions of the under-moderated KJRR element. 

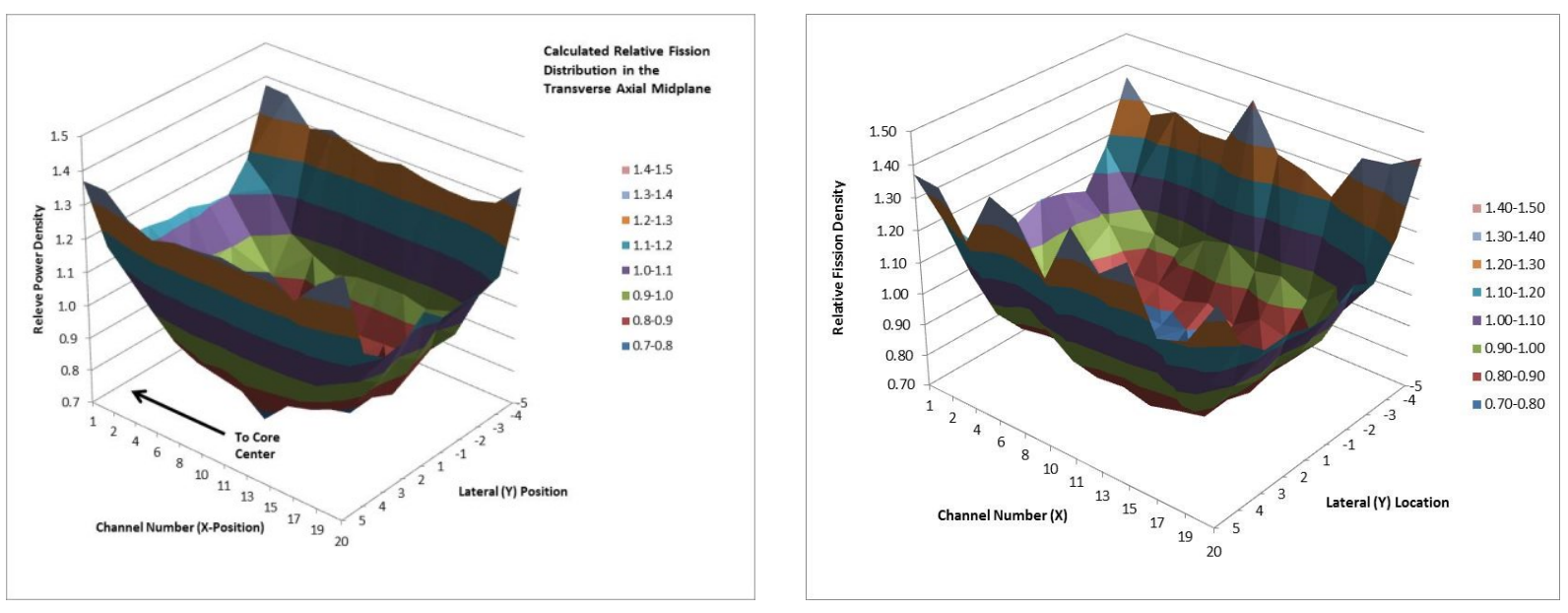

Figure 16. Calculated (Left) and Measured (Right) relative fission distributions in the transverse axial midplane of the KJRR fuel element

The fission rate measurements in the 360 wires located in the KJRR fuel element can also be used to produce experimental estimates of the absolute total power being generated in that element during the 15-2 Irradiation. Two different, somewhat independent, methods for accomplishing this are available:

First, recall that the measured fission rates for the wires are reported in watts per gram of ${ }^{235} \mathrm{U}$ in the wire assuming $197 \mathrm{MeV}$ energy released per fission. The same quantity (after some unit conversions and energy per fission adjustments) can be computed for each wire using the MCNP5 fission tally. Thus, if the MCNP5 calculation is normalized to the reported total reactor power for the irradiation (724.63 Watts) then the calculated fission power in each wire can be compared to the corresponding measured power in an absolute sense. This produces a set of 360 ratios of calculated to measured fission power for the wires in the element. The average of these ratios and its standard deviation then provide an estimate of the bias of the calculated KJRR fuel element power from the MCNP5 model relative to the measurement, along with the statistical distribution of the bias. This approach is based on the assumption that any bias observed in the wire measurements will be fractionally the same as the bias that would be observed for the actual element power if the fission rates within the KJRR fuel plates could have been directly measured. Note that the total ATRC core power is measured by exactly the same type of fission wires as were located in the KJRR fuel element. Furthermore, the fission rates in all of the wires, those in the core fuel elements and those in the KJRR fuel element alike, were measured using the same beta detector system, with the same calibration and the same measurement protocol, so any systematic uncertainties in ratios of fission rates in the wires would tend to cancel.

Table 1 summarizes the results of the above method for estimating the total fission power produced in the KJRR fuel element. The average ratio of calculated to measured wire fission rates for the 360 fission wires in the KJRR fuel element was 1.163 , with a standard deviation of about $4 \%$, which is comparable to the random uncertainty (5\%) in the wire fission rate measurements themselves. The calculated power of the KJRR fuel element was 23.22 Watts. Dividing this by the measured average bias (1.163) of all 360 fission wires in the KJRR fuel element yields a "measured" total element power of 19.96 Watts with a statistical uncertainty in the range of about $4 \%$. Recall that the calculated total power of the fuel elements that comprise the NW lobe of the ATRC was about $8 \%$ higher than the corrected measurement. This accounts for approximately half of the average difference (16\%) between the calculated and measured fission rates in the wires within the KJRR element.

The second method for using the measured fission rates in the wires to estimate the total power of the KJRR fuel element takes an alternate approach that explicitly makes use of detailed MNCP calculations of the wire fission rates relative to the calculated fission rates in the adjacent KJRR fuel plates. This produces a somewhat more direct measured estimate that accounts for the fact that the fission rates in the wires are essentially unaffected by spatial self-shielding [14] whereas the fission rates in the KJRR fuel plates would be expected to exhibit some selfshielding due to the presence of significantly greater quantities of ${ }^{238} \mathrm{U}$ in the KJRR fuel plates relative to the fission wires, which use fully-enriched $\left(93 \%{ }^{235} \mathrm{U}\right)$ uranium. 


\section{[Type here]}

Table 1. Statistical analysis of calculated and measured fission rates for all 360 fission wires in the KJRR fuel element.

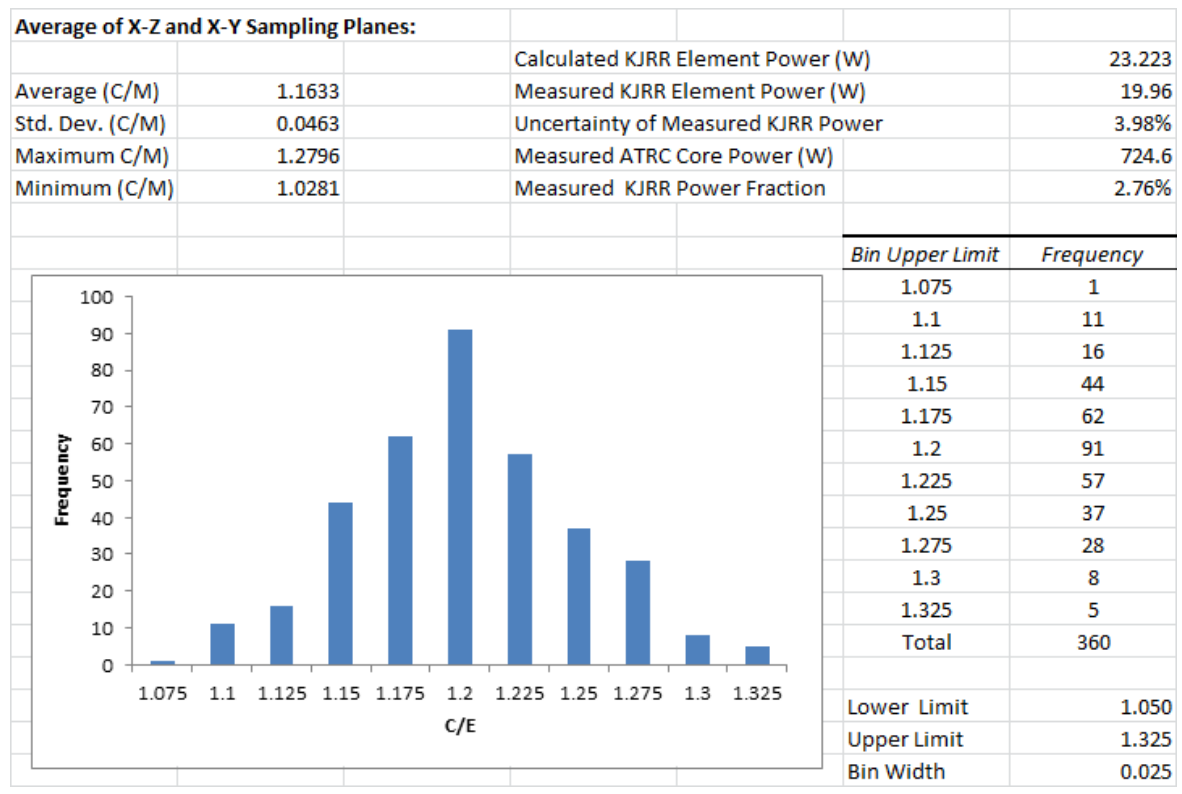

To implement the second approach, recall that Figure 16 (R) shows the relative distribution of fission rates measured for the 132 fission wires located at the axial midplane of the KJRR element. Also recall from Figure 7 that these wires were located in Channels A,B,D,F,H,J,K,M,O,Q,S, and T, while Channels C,E,G,I, L,N, P and R did not contain wires. he fission rates that would have been measured in wires located in the latter 8 channels can be estimated by linear interpolation of the actual measurements for wires on the channels on either side of the channels without wires. Averaging the actual measurements and the interpolated measurements for the wires yields a weighted-average value of 0.0401 watts per gram of ${ }^{235} \mathrm{U}$ for wires located at the axial midplane of the KJRR element. This result is incidentally consistent with the unweighted average of $0.0408 \mathrm{w} / \mathrm{g}$ for only the actual wires. Analysis of the axial fission rate distribution data for the KJRR element yields a transverse average axial power peaking factor for the KJRR fuel element of 1.125. Dividing the average measured power of the fission wires located at the midplane by this peaking factor yields a measured axial average power in the fission wires of 0.0356 watts per gram of ${ }^{235} \mathrm{U}$.

Proceeding further, MCNP5 calculations show that on a similar average basis, the fission rate in the same units (but including fissions of both ${ }^{235} \mathrm{U}$ and a very small contribution from fission of ${ }^{238} \mathrm{U}$ ) at a given location in a KJRR fuel plate is $93.5 \%$ of that in the most immediately adjacent wire, with a standard deviation approximately $5 \%$. Applying this factor to the measured axial average power in the fission wires yields an average power in the KJRR fuel plates of 0.0333 watts per gram of ${ }^{235} \mathrm{U}$ in the plates. Multiplying this by the total mass of ${ }^{235} \mathrm{U}$ in the KJRR plates $(618.3$ g) yields a total power of 20.6 watts for the element, with an uncertainty of about $7 \%$, accounting for the inherent uncertainty of the wire measurements along with the standard deviation of the calculated ratio of plate power relative to adjacent fission wire power. This is in good statistical agreement with the result obtained using the first method outlined above (19.96 watts), but still about 13\% lower than the corresponding a priori calculation (23.22 watts). As noted earlier, about $8 \%$ of the difference between measurement and calculation is attributable to lobe power difference and the remaining $5 \%$ in this case would be due to possible model biases and other sources of uncertainty.

Furthermore, following the procedures outlined in Reference 16, a least-squares adjustment of the a-priori power of the KJRR element in the ATRC using the same methodology and models that were used to produce the core fuel element power distribution information shown in Figure 13, but including the power of the KJRR element in the formulation as if it were another ATRC fuel element yields an adjusted power of 21.25 watts for the KJRR element. This is only about $4.8 \%$ higher than the average of the two corresponding measurements, consistent with the discussion immediately above.

Finally, we note from Reference [13] that the reactivity of the KJRR fuel element in the ATRC NE Flux Trap was somewhat overestimated ( $\$ 0.45)$ by the MCNP5 model. This is consistent with the apparent slight overestimate of the KJRR element power in the MCNP5 calculation compared to the results from the fission wire measurements. Further investigation to identify the source of this discrepancy is warranted, but the apparent difference between 
calculated and measured KJRR fuel element power in the ATRC is neither of a magnitude nor is it in the direction that would put the safety of the planned irradiation of the same KJRR fuel element at power in the ATR into question.

\subsection{EFFECT OF THE KJRR EXPERIMENT ON THE ATRC CORE FUEL INTRA-ELEMENT POWER DISTRIBUTION}

The unusually large quantity of fuel in the NE Flux Trap associated with the KJRR fuel element was expected to perturb the axial power distribution in the surrounding fuel elements to an extent that had not been well-quantified experimentally and validated in the computational models. Accordingly, measurements were made in the low-power ATRC KJRR irradiations to address this issue as well, since the power distribution in the ATR core fuel elements at the high power levels typically used for actual irradiations is a key safety parameter.

As noted earlier, the odd-numbered ATRC core fuel elements were heavily instrumented with fission wires in both of the two KJRR irradiations, 15-1 and 15-2. The fission wires at the axial midplane of the instrumented core fuel elements, arranged as shown in Figure 6, are used to determine the total reactor power as described previously. They also provide a measure of the radial and azimuthal fission rate distribution within each instrumented element. In addition, ATRC Fuel Element 5, which was a standard ATR fuel element instead of an ATRC fuel element was instrumented with fission wires arrayed in the axial direction at the five radial positions along the central rib. Figure 17 provides a direct comparison of the measured axial fission rate distributions in ATRC Core Fuel Element 5 from the two irradiations, averaged over the five radial wire positions. The difference between the two fission rate distributions is readily apparent. Corresponding MCNP5 calculations produce results that are in excellent statistical consistency with the measured results in both cases.

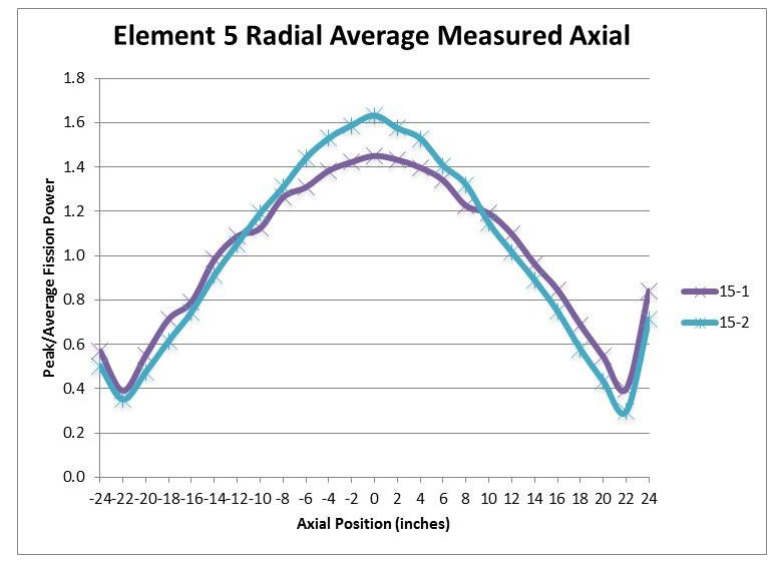

Figure 17. Measured relative wire fission rates for ATRC Irradiations 15-1 and 15-2 as functions of axial (Z) position, averaged over the 5 instrumented channels of ATRC fuel element 5 . Statistical uncertainties $(1 \sigma)$ are in the range of $3 \%$.

\subsection{EFFECT OF THE KJRR EXPERIMENT ON THE ATR LOBE POWER MEASUREMENT SYSTEM}

This section summarizes analyses of some additional measurements taken during ATRC Irradiations 15-1 and 15-2 that were designed to simulate the operation of the ATR Lobe Power Calculation and Indication System (LPCIS). The purpose of the measurements and analyses was to obtain information pertinent to the potential need for recalibration of the LPCIS to support the KJRR irradiation, where the presence of the highly-reactive, partial height, KJRR fuel element in the NEFT was expected to distort the standard relationships between the lobe powers and the signals from the LPCIS neutron flux monitors. The results of the ATRC LPCIS validation measurements and their analysis are summarized, along with an application of the same analytical techniques to the actual LPCIS system in the ATR. 


\section{[Type here]}

\subsection{Description of the ATR LPCIS}

The LIPCIS validation and recalibration measurements were performed by placing $7.62 \mathrm{~cm}$ (3") segments of nickel flux wire in each of the four inboard and two center ATRC N-16 measurement tubes at the axial midplane of the core (Figure 18). The ${ }^{58} \mathrm{Ni}(\mathrm{n}, \mathrm{p})^{58} \mathrm{Co}$ interaction that occurs in these wires when the ATRC is operating is used to simulate the ${ }^{16} \mathrm{O}(\mathrm{n}, \mathrm{p})^{16} \mathrm{~N}$ interaction that occurs in core cooling water flowing through tubes placed in similar positions in the ATR, where the production rate of ${ }^{16} \mathrm{~N}$ in those six positions, along with four additional "outboard" positions, is measured online and subsequently used to deduce the powers of the five ATR 'Lobes" via an overdetermined system of 11 equations that relate the measured nitrogen production rates to the lobe powers of interest

In the case of the ATRC, the fission powers of the fuel elements were independently measured during Irradiations 15-1 and 15-2 by arrays of U/Al fission wires placed within the fuel elements that comprise each of the lobes as described earlier. The lobe powers were deduced by simply adding the powers of the fuel elements associated with each lobe. The results can be compared to lobe powers that are reconstructed from the ATRC nickel wire measurements using the same mathematical methods as are used to reconstruct ATR lobe powers from ${ }^{16} \mathrm{~N}$ production in the ATR. This provides information about the expected behavior of the ATR LPCIS system under various conditions that are simulated in the ATRC. In particular, it was of interest to estimate the extent to which the entries in the coefficient matrix used to define the lobe power reconstruction equations would be perturbed by the presence of the KJRR fuel element in the NEFT.

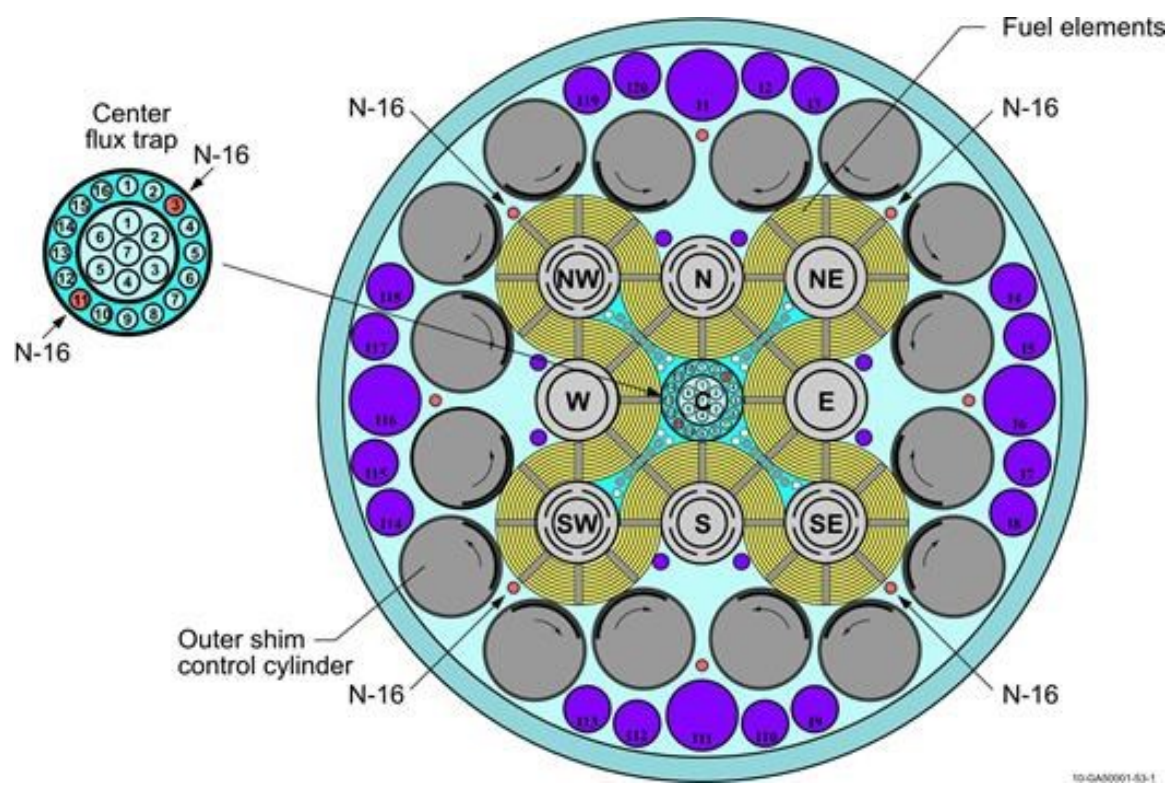

Figure 18. Transverse section of ATRC core showing the simulated N-16 detector locations. Nickel wires were placed in the six locations specifically labeled "N-16". The two locations in the Center Flux Trap are referred to as $\mathrm{H}-3$ and $\mathrm{H} 11$ in the text.

\subsection{Lobe Power Reconstruction Methodology used by the ATR LPCIS}

In the following description of the lobe power reconstruction equations used with the LPCIS system, matrix and vector quantities will generally be indicated by bold typeface. In some cases, matrices and vectors will be enclosed in square brackets for clarity. The superscripts, "-1" and " $\mathbf{T}$ " respectively, indicate matrix inversion and transposition.

Reconstruction of lobe powers from the LPCIS flux monitor signals is accomplished via the following basic overdetermined set of linear equations: 
[Type here]

$$
\left[\begin{array}{lllll}
a_{11} & a_{12} & a_{13} & \cdots & a_{1 N L} \\
a_{21} & a_{22} & a_{23} & \cdots & a_{2 N L} \\
a_{31} & a_{32} & a_{33} & \cdots & a_{3 N L} \\
\vdots & \vdots & \vdots & & \vdots \\
\vdots & \vdots & \vdots & \vdots & \\
a_{N D 1} & a_{N D 2} & a_{N D 3} & & a_{N D N L}
\end{array}\right] \quad\left[\begin{array}{l}
P_{1} \\
P_{2} \\
P_{3} \\
\vdots \\
P_{N L}
\end{array}\right]=\left[\begin{array}{l}
W 1 R_{1} \\
W 2 R_{2} \\
W 3 R_{3} \\
\vdots \\
\vdots \\
W N D R_{N D}
\end{array}\right]
$$

or, more compactly:

$$
[C][P]=[W R]
$$

where NL is the total number of lobes (i.e. 5 for both ATR and ATRC) and ND is the number of flux monitors ("Detectors"). ND is typically 10 for the ATR and it is 6 for the ATRC. In the case of the ATR it is the activation rate of ${ }^{16} \mathrm{O}$ that is measured in each monitor location whereas in the ATRC it is the activation of ${ }^{58} \mathrm{Ni}$ that is measured. The vector $[\mathbf{P}]$ is the desired estimate for the lobe powers and the vector $[\mathbf{W R}]$ contains the ND detector responses, $\mathrm{R}_{\mathrm{i}}$, each multiplied by an arbitrary "Multiplier", $\mathrm{W}_{\mathrm{i}}$, a weight factor that can be used to modify the detector response to compensate for variations of known external factors (e.g. a differing time for coolant transport to the detectors, changing detector efficiencies, etc.) as needed. The matrix [C] contains the coefficients $a_{i, j}$ that relate the response of a given detector, $i$ to the power in a given lobe, $j$. These coefficients may be measured, as was the case for the standard set of coefficients used for routine ATR operations, or they may be calculated by a suitable neutron transport code, as is done using MCNP5 in the work described here.

In practice, an extra equation is also included in the system to force the sum of the reconstructed lobe powers to match an independently-measured value of the total reactor power:

$$
\mathrm{S}\left(\mathrm{P}_{1}+\mathrm{P}_{2} \ldots \ldots \ldots+\mathrm{P}_{\mathrm{NL}}\right)=\mathrm{SP}_{\mathrm{T}}
$$

where $\mathrm{S}$ is a scaling factor that can be set to zero to allow the total power to be unconstrained, or increased to a value that weights the additional equation to a sufficient degree to force the sum of the lobe powers to match the desired total power to a reasonable number of significant figures, but without putting so much weight on the summation equation that numerical instabilities are introduced in the solution for the individual lobe powers because of the finite precision of machine computations.

Combining Equations 2 and 3 yields

$$
[\mathbf{A}][\mathbf{P}]=[\mathbf{Z}]
$$

where the last row of the matrix [A] and the last entry in the vector [Z] are used to add Equation 3 to the unconstrained system represented by Equations 1 and 2.

The augmented system of Equations (4) can be solved by the standard inverse covariance-weighted least-square approach represented by the "Normal Equations" [17]:

$$
B P=A^{T}[\operatorname{Cov}(Z)]^{-1} Z
$$


where $[\operatorname{Cov}(\mathbf{Z})]$ is a covariance matrix associated with the measured detector responses and the measured total reactor power and

$$
\mathbf{B}=\mathbf{A}^{\mathrm{T}}[\operatorname{Cov}(\mathbf{Z})]^{-1} \mathbf{A}
$$

Also, since the solution to Equation 5 is:

$$
\mathbf{P}=\mathbf{B}^{-1} \mathbf{A}^{\mathrm{T}}[\operatorname{Cov}(\mathbf{Z})]^{-1} \mathbf{Z}
$$

the covariance matrix for the lobe powers may be computed by the standard uncertainty propagation formula:

$$
[\operatorname{Cov}(P)]=D \operatorname{Cov}(Z) D^{T}
$$

where

$$
\mathbf{D}=\mathbf{B}^{-1} \mathbf{A}^{\mathrm{T}}[\operatorname{Cov}(\mathrm{Z})]^{-1}
$$

The diagonal elements of the covariance matrix for the adjusted powers can then be used to estimate the uncertainties of the lobe powers due to propagated uncertainties of the detector responses if desired. Finally, it is important to note that in the work presented here the detector signal and total power covariance matrix $[\mathbf{C o v}(\mathbf{Z})]$ in the derivation above was simply set equal to an identity matrix in Equation 5 to match the current programming of the LPCIS system.

\subsection{Measurement Protocol for the ATRC}

As noted earlier, two ATRC Irradiations were completed to support the KJRR experiment campaign, one with the KJRR fuel element and its inner basket positioned in the NEFT (15-2) and the other without (15-1). In the latter case the space that would ordinarily be occupied by the KJRR element and its inner basket was filled with roomtemperature water from the ATRC pool. Further details of the two ATRC configurations are provided in previous sections. In each irradiation, small segments of natural abundance nickel flux wire, $76.2 \mathrm{~mm}$ (3") in length, with an average mass of $277 \pm 1 \mathrm{mg}$ each were placed at the core axial midplane in each of the four inboard and the two center detector positions indicated in Figure 18. Note that Figure 18 also shows 4 additional detector positions at 3, 6,9 , and 12 o'clock at the approximately same distance from the core center as is the case for the four labeled N-16 positions adjacent to the NE, SE, SW, and NW lobes. These so-called "outboard" positions are ordinarily used in the ATR but not in the ATRC. Thus the system of equations (3) is of dimension $7 \times 5$ for the ATRC, including the total power normalization equation.

The two ATRC irradiations of interest were each 20 minutes in length. At the end of each irradiation the nickel wires were retrieved and sent to the INL RML for gamma spectrometry using HPGe spectrometer systems calibrated to NIST-traceable standards. The induced activities of ${ }^{58} \mathrm{Co}$, corrected to the time of reactor shutdown were reported for each wire. These activities were converted to saturation activity, or equivalently, activation rate, in units of Bq per atom of ${ }^{58} \mathrm{Ni}$ in the wire for each wire using the standard formula:

$$
\sigma \phi_{i}=R_{i}=A_{i} /\left(N_{p}\left(1-\exp \left(-\lambda t_{a}\right)\right)\right)
$$

where $\mathrm{R}_{\mathrm{i}}$ is the saturation activity, or activation rate, in units of $\mathrm{Bq} /$ atom, $\sigma$ is the spectrum-averaged cross section $\left(\mathrm{cm}^{2}\right)$ for the ${ }^{58} \mathrm{Ni}(\mathrm{n}, \mathrm{p})^{58} \mathrm{Co}$ interaction, $\phi$ is the scalar neutron flux (neutrons $/ \mathrm{cm}^{2}-\mathrm{s}$ ) in the wire, integrated over all neutron energies above the threshold for the interaction, $\mathrm{A}_{\mathrm{i}}$ is the measured total activity of the wire $(\mathrm{Bq}$ at reactor shutdown), $\mathrm{N}_{\mathrm{p}}$ is the total number of ${ }^{58} \mathrm{Ni}$ atoms in the wire, calculated from the reported mass of the wire in the usual manner, $\lambda$ is the decay constant for ${ }^{58} \mathrm{Co}\left(\mathrm{s}^{-1}\right)$ and $\mathrm{t}_{\mathrm{a}}$ is the activation time $(1200 \mathrm{~s})$. Note that the saturation activity per parent atom can also be directly computed by MCNP5 using an F4 type tally for the modeled wire 


\section{[Type here]}

volume. In the case of the computations reported here, the cross section data used for tallies of this type were taken from the International Reactor Dosimetry File (IRDF) cross-section library [18].

Figures 19 and 20 respectively show the cross sections for the ${ }^{58} \mathrm{Ni}(\mathrm{n}, \mathrm{p}){ }^{58} \mathrm{Co}$ interaction used in the ATRC LPCIS simulation measurements and for the ${ }^{16} \mathrm{O}(\mathrm{n}, \mathrm{p})^{16} \mathrm{~N}$ interaction that is measured in the ATR LPCIS. Note that the cross sections have roughly the same peak magnitude $(100 \mathrm{mb})$ but the energy threshold in the case of the ${ }^{16} \mathrm{O}$ cross section is approximately $10 \mathrm{MeV}$, where it is only about $1 \mathrm{Mev}$ for ${ }^{58} \mathrm{Ni}$. Thus both cross sections are primarily sensitive to neutrons having energies in the fission source energy range, as desired, but the magnitude of the ${ }^{58} \mathrm{Ni}$ interaction rate per atom will be much greater in a given fission neutron spectrum because of the lower threshold. In fact it would be very difficult to measure the nitrogen activation rate in the ATRC due to the much lower power in the ATRC as well as the fact that the ATRC does not have a system to extract water from the detector locations and send it to a suitable beta detector in a time that is reasonable compared to the 7 second half-life of the ${ }^{16} \mathrm{~N}$ activation product. Furthermore, even if there were such a system in the ATRC it likely would not be possible to obtain a statistically useful number of counts in the detector before all of the ${ }^{16} \mathrm{~N}$ had decayed away. Hence the ${ }^{58} \mathrm{Ni}(\mathrm{n}, \mathrm{p}){ }^{58} \mathrm{Co}$ interaction rate, which is relatively easy to measure to good precision using standard techniques, is employed to simulate the LPCIS detector responses in the ATRC.

It also should be noted that the nickel activation rate in the ATRC will include the effect of photoneutrons produced in the beryllium reflector and moderator regions of the ATRC and ATR cores. However, the threshold for photoneutron production in $\mathrm{Be}$ is $1.66 \mathrm{MeV}$ and, as noted above, the threshold for the ${ }^{58} \mathrm{Ni}(\mathrm{n}, \mathrm{p})^{58} \mathrm{Co}$ interaction is about $1 \mathrm{MeV}$, for a combined effective gamma threshold energy of about $2.7 \mathrm{MeV}$ for activation of Nickel by photoneutrons. Furthermore, the photoneutron cross section for Be is less than $1 \mathrm{mb}$ in the energy range of practical interest $(<10 \mathrm{MeV})$ in this application. Thus the photoneutron effect was determined to be small, and in any event it would tend to cancel to some extent in the coefficient ratios of primary importance in this study, so it was not explicitly included in the calculational models.

The lobe powers were obtained by a simple summation of the powers measured as described previously for the fuel elements associated with each lobe. Thus it is possible to simulate reconstruction of lobe powers in the ATRC using nickel wire measurements in concert with Equations 1-10, and the results can be compared with MCNP5 computations as well as with independently-measured ATRC lobe powers based on the fission wires. In turn, this provides information pertinent to the anticipated accuracy of the LPCIS system as well as information regarding whether the coefficient matrix on the left-hand side of Equation 1 changes significantly when the KJRR fuel element is inserted into the NEFT relative to the case with a water-filled flux trap. Given this validation information from the ATRC it is then possible to make statements about how the LPCIS system is likely to behave in the ATR upon the introduction of such a highly-reactive experimental fuel element into the NEFT.

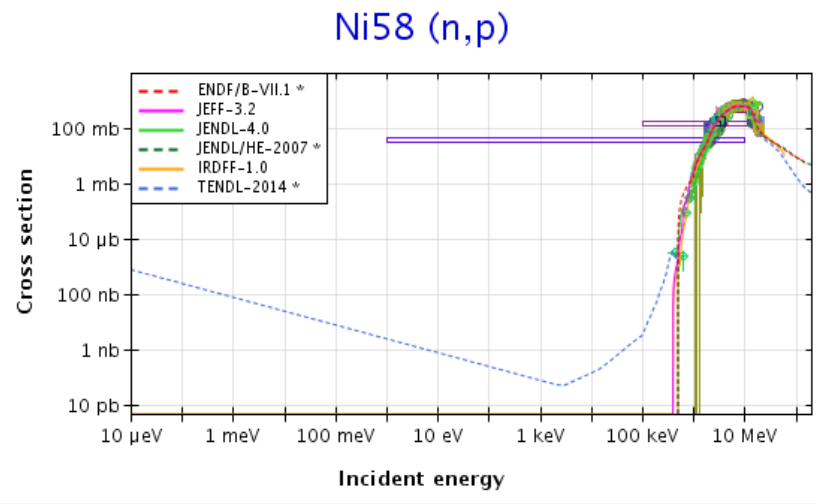

Figure 19. Activation cross section for the ${ }^{58} \mathrm{Ni}(\mathrm{n}, \mathrm{p}){ }^{58} \mathrm{Co}$ interaction (Source: OECD-NEA Janis File, Version 4) 


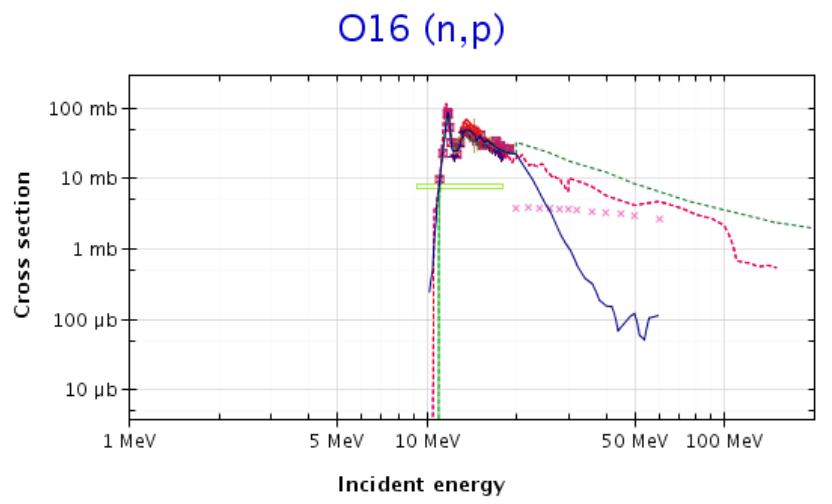

Figure 20. Activation cross section for the ${ }^{16} \mathrm{O}(\mathrm{n}, \mathrm{p}){ }^{16} \mathrm{~N}$ interaction (Source: OECD-NEA Janis File, Version 4)

\subsection{Results}

The following sections first provide the results for the analysis of the ATRC LPCIS simulation measurements for Irradiations 15-1 and 15-2, respectively. The results of calculations performed to apply the concepts developed in the ATRC to the ATR for the full-power KJRR experiment are then summarized.

\section{ATRC Irradiation 15-1}

Table 2 shows the coefficient matrix for the simulated ATRC LPCIS system, computed for Irradiation 15-1 using MCNP5. Each entry is the computed ${ }^{58} \mathrm{Co}$ saturation activity (Bq per ${ }^{58} \mathrm{Ni}$ atom) for the indicated "Detector" (nickel wire) per unit power (Watts) of the indicated core lobe. Table 2 also shows the MCNP5 estimated tally uncertainties for the non-zero entries. Note that the coefficient matrices for the ATRC and ATR computations discussed here were constructed by eliminating entries whose contributions were less $1 \%$ of the maximum coefficient. This simplifies the system of equations for the simulated LPCIS system (Equation 1) without significant loss of precision, and it eliminates coefficients that have high tally uncertainties because they are so small compared to the others. The large relative tally uncertainties for these small coefficients are primarily due to the large fast-neutron attenuation factors associated with their positions in the coefficient matrix, along with the small wire volume and the very small interaction cross section that applies to all of the matrix positions, coupled with the relatively limited number of neutron histories that as a practical matter could be processed in a given MCNP5 calculation.

The reconstructed lobe powers obtained by solving Equation 5 for Irradiation 15-1 using the reduced coefficient matrix and the computed detector responses (nickel wire saturation activities per atom) from the model were within $1 \%$ of the lobe powers that result if the full coefficient matrix is used instead of the reduced matrix. Note that if the full matrix is used for the solution for the reconstructed powers, as opposed to the reduced matrix described, then the results for the powers are identically equal to the original lobe powers computed by MCNP5, since it is the MCNP5 solution that is used to compute the coefficients and detector responses in the first place, so everything balances perfectly, to machine precision. This provides a method to verify software used to implement a simulated LPCIS system model such as the one described here. And it can be seen that use of the reduced matrix considerably simplifies the equations, avoids unnecessarily propagated uncertainty, and still reproduces the a priori lobe powers to well within the numerical tally uncertainties.

The "Total Power" row of Table 2 represents the coefficients that correspond to the $7^{\text {th }}$ equation in the simulated LPCIS system (Equation 3), which forces the total power of the 5 lobes to equal the measured total power of the reactor (472.7 watts). As discussed earlier, the $7^{\text {th }}$ equation is weighted to make it dominate the others, but not so much so that numerical precision errors appear in the machine-computed solution for the individual lobe powers. In the case of Irradiation 15-1, a scaling factor of 1.0E-17 for Equation 3 was found to force the desired total power to an accuracy of four significant figures without creating numerical problems in the overall solution to the coupled equations. 
Table 2. Reduced lobe power reconstruction coefficient matrix entries for Irradiation 15-1 and corresponding MCNP tally uncertainties $(1 \sigma)$. Units are $\mathrm{Bq} /$ atom per unit lobe power in watts.

\begin{tabular}{|c|c|c|c|c|c|}
\hline \multicolumn{6}{|c|}{ Coefficients } \\
\hline Detector & NW Lobe & NE Lobe & SE Lobe & SW Lobe & Center Lobe \\
\hline NW & $2.316 \times 10^{-19}$ & & & & \\
\hline $\mathrm{NE}$ & & $2.356 \times 10^{-19}$ & & & \\
\hline $\mathrm{SE}$ & & & $2.504 \times 10^{-19}$ & & \\
\hline SW & & & & $3.077 \times 10^{-19}$ & \\
\hline $\mathrm{H} 3$ & $5.611 \times 10^{-20}$ & $1.242 \times 10^{-19}$ & $4.208 \times 10^{-20}$ & $1.758 \times 10^{-20}$ & $5.296 \times 10^{-19}$ \\
\hline H11 & $5.185 \times 10^{-20}$ & $1.781 \times 10^{-20}$ & $4.991 \times 10^{-20}$ & $1.027 \times 10^{-19}$ & $7.159 \times 10^{-19}$ \\
\hline Total Power & $1.000 \times 10^{-17}$ & $1.000 \times 10^{-17}$ & $1.000 \times 10^{-17}$ & $1.000 \times 10^{-17}$ & $1.000 \times 10^{-17}$ \\
\hline \multicolumn{6}{|c|}{ Tally Uncertainties (\%) } \\
\hline Detector & NW Lobe & NE Lobe & SE Lobe & SW Lobe & Center Lobe \\
\hline NW & 2.96 & & & & \\
\hline $\mathrm{NE}$ & & 3.33 & & & \\
\hline SE & & & 2.35 & & \\
\hline SW & & & & 1.51 & \\
\hline $\mathrm{H} 3$ & 5.70 & 4.03 & 5.28 & 6.01 & 8.15 \\
\hline H11 & 5.82 & 9.81 & 4.85 & 2.56 & 8.10 \\
\hline
\end{tabular}

With the model verification demonstrated as described above, the actual measured nickel wire activation rates for Irradiation 15-1 can now be substituted into the simulated LPCIS equations, again with the reduced coefficient matrix, to produce the results shown in Table 3, where the reconstructed lobe powers are compared to the lobe powers from the fission wire measurements. Uncertainties for the reconstructed lobe powers are estimated to be in the range of 3\% for the corner lobes and approximately $8 \%$ for the center lobe, given the coefficient tally uncertainties provided in Table 2 . The uncertainty of the directly-measured lobe powers is conservatively taken as $5 \%(1 \sigma)$. This corresponds to the standard accepted uncertainty of the individual measured fuel element powers [14]. Thus the uncertainties $(1 \sigma)$ in the ratios of reconstructed power to measured power range from about $6 \%$ to about $10 \%$, so the reconstructed lobe powers are statistically consistent with the measurements to a reasonable degree.

Table 3. Reconstructed lobe powers (Watts) for Irradiation 15-1 using reduced coefficient matrix and RML measured detector responses.

\begin{tabular}{|c|c|c|c|}
\hline Lobe & $\begin{array}{c}\text { Reconstructed } \\
\text { Power (W) }\end{array}$ & $\begin{array}{c}\text { Measured } \\
\text { Power (W) }\end{array}$ & Ratio R/M \\
\hline NW & 53.569 & 54.414 & 0.984 \\
\hline NE & 57.854 & 54.906 & 1.054 \\
\hline SE & 91.069 & 90.106 & 1.011 \\
\hline SW & 193.116 & 180.540 & 1.070 \\
\hline Center & 77.100 & 92.742 & 0.831 \\
\hline Total & 472.708 & 472.708 & 1.000 \\
\hline
\end{tabular}




\section{[Type here]}

It is also possible to apply "Multipliers" to the measured wire activities in Equation 1 to tune the reconstructed powers to better match the directly measured lobe powers if this is deemed advisable because of a known physical situation causing the detectors to behave differently than was the case when the LPCIS system was most recently calibrated. The use of the multipliers to modify the solution of the LPCIS reconstruction equations for small changes in known system parameters is illustrated numerically here by iterating a vector of trial multipliers until the reconstructed lobe powers match the measured powers to a desired degree Table 4 shows a resulting example set of multipliers that can be used to modify the measured nickel wire activities in the Irradiation 15-1 simulation to demonstrate this process (note that the multipliers were all 1.00 to produce the unmodified lobe power reconstruction results shown in Table 3). Table 5 shows the reconstructed lobe powers with the multipliers applied. It can be seen that the reconstructed powers can be brought into approximately $1 \%$ agreement with a set of multipliers that differ from 1.0 only by amounts that are reasonably consistent with the combined propagated uncertainties discussed earlier.

Table 4. Example detector response multipliers for Irradiation 15-1

\begin{tabular}{|c|c|c|}
\hline Detector & Multiplier & $\begin{array}{c}\text { Modified } \\
\text { Detector } \\
\text { Response }\end{array}$ \\
\hline NW & 1.180 & $1.345 \times 10^{-17}$ \\
\hline NE & 1.100 & $1.351 \times 10^{-17}$ \\
\hline SE & 1.050 & $2.300 \times 10^{-17}$ \\
\hline SW & 0.950 & $5.597 \times 10^{-17}$ \\
\hline H3 & 1.140 & $6.731 \times 10^{-17}$ \\
\hline H11 & 1.140 & $9.401 \times 10^{-17}$ \\
\hline
\end{tabular}

Table 5. Reconstructed lobe powers (Watts) for Irradiation 15-1 using reduced coefficient matrix and RML measured detector responses with multipliers from Table 4 applied.

\begin{tabular}{|c|c|c|c|}
\hline Lobe & $\begin{array}{c}\text { Reconstructed } \\
\text { Power (W) }\end{array}$ & $\begin{array}{c}\text { Measured } \\
\text { Power (W) }\end{array}$ & Ratio R/M \\
\hline NW & 54.812 & 54.414 & 1.007 \\
\hline NE & 55.010 & 54.906 & 1.002 \\
\hline SE & 88.952 & 90.106 & 0.987 \\
\hline SW & 179.714 & 180.540 & 0.995 \\
\hline Center & 94.225 & 92.742 & 1.016 \\
\hline Total & 472.712 & 472.708 & 1.000 \\
\hline
\end{tabular}

\section{ATRC Irradiation 15-2}

We now turn to Irradiation 15-2, where the KJRR fuel element and its inner basket were present in the NE LIPT, displacing most of the water that was in the fuel element and inner basket regions during Irradiation 15-1. In the analyses for this case it is assumed for both computational and experimental purposes that the "Lobe Power" for the NE Lobe includes the fission power of the KJRR fuel element in addition to the power of ATRC core fuel elements $2-9$, in this case adding about $10 \%-12 \%$ to the traditionally-defined lobe power.

Table 6 shows the reconstructed powers for the case where the detector responses measured for Irradiation 2 are used with the reduced coefficient matrix, also for Irradiation 2, computed by MCNP5. Finally, Tables 7 and 8 


\section{[Type here]}

demonstrate how the reconstructed lobe powers obtained in Table 6 can be adjusted back into better agreement with the directly measured powers through the use of suitable multipliers.

Table 6. Reconstructed Lobe Powers (Watts) for Irradiation 15-2 using reduced coefficient matrix and RML measured detector responses

\begin{tabular}{|c|c|c|c|}
\hline Lobe & $\begin{array}{c}\text { Reconstructed } \\
\text { Power (W) }\end{array}$ & $\begin{array}{c}\text { Measured } \\
\text { Power (W) }\end{array}$ & Ratio R/M \\
\hline NW & 98.276 & 79.060 & 1.243 \\
\hline NE & 184.604 & 189.749 & 0.973 \\
\hline SE & 129.868 & 127.700 & 1.017 \\
\hline SW & 199.157 & 196.660 & 1.013 \\
\hline Center & 132.991 & 151.740 & 0.876 \\
\hline Total & 744.896 & 744.909 & 1.00 \\
\hline $\begin{array}{l}\text { Note: Power for the NE Lobe includes the power of the KJRR } \\
\text { fuel element. }\end{array}$ \\
\hline
\end{tabular}

Table 7. Example detector response multipliers for Irradiation 15-2

\begin{tabular}{|c|l|c|}
\hline Detector & Multiplier & $\begin{array}{c}\text { Modified } \\
\text { Detector } \\
\text { Response }\end{array}$ \\
\hline $\mathrm{NW}$ & 0.700 & $1.046 \times 10^{-17}$ \\
\hline $\mathrm{NE}$ & 1.025 & $5.702 \times 10^{-17}$ \\
\hline $\mathrm{SE}$ & 0.970 & $2.470 \times 10^{-17}$ \\
\hline $\mathrm{SW}$ & 0.970 & $4.702 \times 10^{-17}$ \\
\hline $\mathrm{H} 3$ & 1.090 & $1.290 \times 10^{-16}$ \\
\hline $\mathrm{H} 11$ & 1.090 & $1.352 \times 10^{-16}$ \\
\hline
\end{tabular}

Table 8. Reconstructed Lobe Powers (Watts) for Irradiation 15-2 using reduced coefficient matrix and RML measured detector responses with multipliers from Table 7 applied.

\begin{tabular}{|c|c|c|c|}
\hline Lobe & $\begin{array}{c}\text { Reconstructed } \\
\text { Power (W) }\end{array}$ & $\begin{array}{c}\text { Measured } \\
\text { Power (W) }\end{array}$ & Ratio R/M \\
\hline NW & 79.387 & 79.060 & 1.004 \\
\hline NE & 189.314 & 189.749 & 0.998 \\
\hline SE & 128.507 & 127.700 & 1.006 \\
\hline SW & 196.205 & 196.660 & 0.998 \\
\hline Center & 151.481 & 151.740 & 0.998 \\
\hline Total & 744.895 & 744.909 & 1.000 \\
\hline $\begin{array}{l}\text { Note: Power for the NE Lobe includes the power of the KJRR } \\
\text { fuel element. }\end{array}$
\end{tabular}




\section{[Type here]}

Moving now to Table 9, we examine the ratios of the reduced coefficient matrix entries for Irradiation 15-2 relative to the corresponding entries for Irradiation 15-1, along with the estimated tally uncertainties for the ratios. These ratios (within their statistical uncertainties) provide measures of the distortion of the coefficient matrix caused by the presence of the KJRR fuel element in the NE lobe. Of particular interest is the entry for the effect of the NE lobe power on the immediately-adjacent NE detector (nickel wire). The coefficient increases by about $34 \%$ in the case of this lobe. Part of this increase is due to the fact that the nickel wires are located at the axial midplane of the ATRC core, where the measured axial fission power peaking factor in the NE lobe near the NE detector wire is 1.63 in Irradiation 15-2 as opposed to the usual value of 1.42 that was measured in the case of Irradiation 15-1. This alone causes a coefficient change of nearly $15 \%$ upward for the case with the KJRR fuel element present in the ATRC NE lobe. It should be noted however that this axial effect would not be present in the water-filled ${ }^{16} \mathrm{~N}$ detector regions of the ATR since the water in the detector flow tubes is exposed to the entire axial length of the core as it flows through them, tending to average out any axial peaking effects, all other factors being equal.

A second plausible reason for the large change observed for the NE detector coefficient in the second column of Table 9 has to do with the fact that the ATRC irradiations were specifically designed to explore the entire anticipated range of coefficient shift magnitudes. In particular, the water-filled condition of the NE flux trap in Irradiation 15-1 represents a bounding case in the sense that fission neutrons originating in the core fuel elements in the NE lobe in this case are much more likely to be scattered down in energy to the point where they cannot activate the nearby detector than would be the case in the more usual situation where the NEFT contains a low-specificactivity cobalt irradiation hardware assembly (LSA Cobalt) that is largely ( $>50 \%$ by volume) composed of metal, as in the ATR. Presence of a large volume of metal in the NEFT would significantly increase the baseline NE detector coefficient compared to the case with a water-filled NEFT.

Table 10 shows the effect of using the reduced coefficient matrix for Irradiation 15-1 and the computed detector responses for Irradiation 15-2 in Equation 5 to compute the lobe power vector. It can be seen that fairly large errors are introduced by the coefficient shift, and therefore it is not particularly appropriate to use the coefficients for Irradiation 15-1 to reconstruct lobe powers for Irradiation 15-2, although even here the errors could be compensated by applying multipliers to the detector responses.

Table 9. Ratio of reduced lobe power reconstruction coefficient matrix entries for Irradiation 15-2 relative to the reduced coefficient matrix entries for Irradiation 15-1.

\begin{tabular}{|c|c|c|c|c|c|}
\hline \multicolumn{6}{|c|}{ Coefficient Ratios (Irradiation 15-2/Irradiation 15-1) } \\
\hline Detector & NW Lobe & NE Lobe & SE Lobe & SW Lobe & Center Lobe \\
\hline NW & 0.913 & & & & \\
\hline $\mathrm{NE}$ & & 1.335 & & & \\
\hline SE & & & 0.944 & & \\
\hline SW & & & & 0.946 & \\
\hline $\mathrm{H} 3$ & 0.987 & 0.924 & 1.243 & 0.946 & 1.243 \\
\hline H11 & 0.993 & 1.221 & 0.989 & 1.012 & 0.881 \\
\hline \multicolumn{6}{|c|}{ Tally Uncertainties (\%) } \\
\hline Detector & NW Lobe & NE Lobe & SE Lobe & SW Lobe & Center Lobe \\
\hline NW & 6.41 & & & & \\
\hline $\mathrm{NE}$ & & 4.04 & & & \\
\hline SE & & & 4.35 & & \\
\hline SW & & & & 3.11 & \\
\hline H3 & 10.44 & 5.40 & 9.04 & 12.84 & 12.41 \\
\hline H11 & 10.42 & 13.31 & 9.18 & 4.83 & 12.29 \\
\hline
\end{tabular}




\section{[Type here]}

Table 10. Comparison of reconstructed lobe powers (Watts) for Irradiation 15-2 using reduced coefficient matrices for Irradiation 15-1 and Irradiation 15-2 with the the computed detector responses for Irradiation 15-2 in both cases.

\begin{tabular}{|c|c|c|c|}
\hline Lobe & $\begin{array}{l}\text { Reconstructed Power (W) } \\
\text { Based On Irradiation 15-1 } \\
\text { Coefficients }\end{array}$ & $\begin{array}{l}\text { Reconstructed Power (W) } \\
\text { Based on Irradiation 15-2 } \\
\text { Coefficients }\end{array}$ & $\begin{array}{l}\text { Ratio of Results with 15-1 } \\
\text { Coefficients to Results with 15-2 } \\
\text { Coefficients }\end{array}$ \\
\hline NW & 64.655 & 83.663 & 0.773 \\
\hline NE & 280.472 & 205.311 & 0.836 \\
\hline SE & 101.892 & 121.918 & 0.807 \\
\hline SW & 150.645 & 186.767 & 1.000 \\
\hline Center & 147.256 & 147.252 & \\
\hline
\end{tabular}

To illustrate the use of multipliers to compensate for coefficient shifts in this case, Table 11 shows an example of a set of multipliers for the computed detector responses that forces the reconstructed lobe powers to be consistent with the computed lobe powers for Irradiation 15-2 (the "Target Powers") even though the coefficient matrix for Irradiation 15-1 is used for the reconstruction. The results of applying these multipliers are shown in Table 12.

Table 11. Example Multipliers to force 15-2 reconstructed powers using 15-1 coefficients to match 15-2 computed powers.

\begin{tabular}{|c|c|c|}
\hline Detector & Multiplier & $\begin{array}{c}\text { Modified } \\
\text { Detector } \\
\text { Response }\end{array}$ \\
\hline NW & 1.05 & $1.870 \times 10^{-17}$ \\
\hline NE & 0.63 & $4.068 \times 10^{-17}$ \\
\hline SE & 1.05 & $3.015 \times 10^{-17}$ \\
\hline SW & 1.18 & $6.028 \times 10^{-17}$ \\
\hline H3 & 1.00 & $1.347 \times 10^{-16}$ \\
\hline H11 & 1.00 & $1.272 \times 10^{-16}$ \\
\hline
\end{tabular}

Table 12. Adjusted powers computed using Irradiation 15-1 coefficients and multipliers from Table 11 with the computed detector responses for Irradiation 15-2.

\begin{tabular}{|c|c|c|c|}
\hline Lobe & $\begin{array}{c}\text { Reconstructed Power (W) } \\
\text { Based On Irradiation 15-1 } \\
\text { Coefficients }\end{array}$ & Target Power (W) & $\begin{array}{c}\text { Ratio of Reconstructed Power to } \\
\text { Target Power }\end{array}$ \\
\hline NW & 85.042 & 83.663 & 1.016 \\
\hline NE & 205.519 & 205.311 & 0.990 \\
\hline SE & 120.644 & 121.918 & 0.988 \\
\hline SW & 184.599 & 186.767 & 1.013 \\
\hline Center & 140.107 & 147.252 & \\
\hline
\end{tabular}




\section{[Type here]}

Finally, Tables 13 and 14 illustrate the use of multipliers to modify the measured detector responses for Irradiation 15-2 so that the reconstructed lobe powers for Irradiation 15-2 obtained using the Irradiation 15-1 coefficient matrix are consistent with the directly measured lobe powers for Irradiation 15-2. Once again, consistency can be obtained, but fairly large adjustments to the detector responses, using multipliers, are necessary if the coefficient matrix is not updated to be consistent with the physical configuration of the reactor for Irradiation 15-2.

Table 13. Example Multipliers applied to measured detector responses for Irradiation 15-2 to force agreement of reconstructed powers and RML measured powers when 15-1 coefficients are used for the reconstruction.

\begin{tabular}{|c|c|c|}
\hline Detector & Multiplier & $\begin{array}{c}\text { Modified } \\
\text { Detector } \\
\text { Response }\end{array}$ \\
\hline NW & 0.75 & $1.120 \times 10^{-17}$ \\
\hline NE & 0.60 & $3.337 \times 10^{-17}$ \\
\hline SE & 1.00 & $2.546 \times 10^{-17}$ \\
\hline SW & 1.18 & $5.720 \times 10^{-17}$ \\
\hline H3 & 1.08 & $1.278 \times 10^{-16}$ \\
\hline H11 & 1.08 & $1.340 \times 10^{-16}$ \\
\hline
\end{tabular}

Table 14. Reconstructed lobe powers for Irradiation 15-2 using Irradiation 15-1 coefficients and multipliers shown in Table 13 for modification of measured detector responses from Irradiation 15-2.

\begin{tabular}{|c|c|c|c|}
\hline Lobe & $\begin{array}{c}\text { Reconstructed } \\
\text { Power (W) }\end{array}$ & $\begin{array}{c}\text { Measured } \\
\text { Power (W) }\end{array}$ & Ratio R/M \\
\hline NW & 80.028 & 79.060 & 1.012 \\
\hline NE & 190.133 & 189.749 & 1.002 \\
\hline SE & 126.839 & 127.700 & 0.993 \\
\hline SW & 194.670 & 196.660 & 0.990 \\
\hline Center & 153.223 & 151.740 & 1.010 \\
\hline Total & 744.894 & 744.909 & 1.000 \\
\hline $\begin{array}{l}\text { Note: Power for the NE Lobe includes the power of the KJRR } \\
\text { fuel element. }\end{array}$ \\
\hline
\end{tabular}

The various exercises and examples shown for the ATRC measurements demonstrate that the KJRR fuel element distorts the simulated LPCIS lobe power reconstruction coefficients for the ATRC to a sufficient degree to warrant a concern about whether the distortion would be similar in the ATR, thereby causing unacceptable levels of bias in the reconstructed ATR lobe powers. The ATRC measurements were designed to provide information regarding an anticipated bounding case where the magnitudes of the shifts in coefficients from the base case and the case with the KJRR fuel element installed were intentionally maximized. If similar behavior extends to the case with the ATR, even to a somewhat smaller degree, then the question arises as to whether the ATR LPCIS system should be retuned using multipliers in some manner in order to reduce or remove the bias. Alternatively, it raises the question as to whether it might be more prudent to actually change the coefficients used in the LPCIS software. The numerical analyses described below apply the methodologies outlined in the previous two sections to develop information that can help to answer these questions. 


\section{Application of the LPCIS simulation and analysis methodology to ATR Cycles 157D and $158 \mathrm{~A}$}

This section presents some numerical modeling of the ATR LPCIS system using the same methodology described and verified in the previous two sections to investigate the potential for significant coefficient distortions caused by the presence of the KJRR element in the NEFT of the ATR during the first planned operating cycle with the KJRR fuel element in place (Cycle 158A). The observed distortions are evaluated relative to the configuration of the reactor as it was for the previous cycle (157D) where the NEFT contained an array of targets used for routine production of ${ }^{60} \mathrm{Co}$.

In addition to the LPCIS, the ATR has an independent calorimetric system (the so-called "water power calculator") that measures the four ATR "quadrant powers", which are directly related to the five ATR lobe powers. The Northeast Quadrant Power for example consists of the NE lobe power (powers of Fuel Elements 2-9 plus the KJRR fuel element power in the case of Cycle 158A) plus the powers of Fuel Elements 1 and 10 in the center lobe (approximated as $25 \%$ of the total Center Lobe power). It may be noted here that since the quadrant powers measured in the ATR by the Water Power Calculator would automatically include the effect of the energy added to the coolant by fissions occurring in the KJRR fuel element, the assumption of including the KJRR power in the NE lobe power reconstruction in the LPCIS equations as discussed earlier is viewed as scientifically consistent and reasonable. During ATR startup, the quadrant powers measured by the Water Power Calculator must be in agreement with the quadrant powers constructed from the LPCIS measurements to within approximately $8 \%$, or ascension to full power is not permitted.

As before, the MCNP5 code was used for the modeling. Beginning of Cycle (BOC) models of the configurations of the ATR for Cycles 157D and 158A at power, with the rotating shims set to the actual positions that produced the desired lobe power splits, were assembled using the baseline MCNP5 production model of the ATR. In both cycles, the core fuel loading configuration included both fresh and partially-burned fuel elements per the loading specifications for each cycle. All flux traps and other experiment positions were represented in the models with their actual (Cycle 157D) contents in both cases.

In these simulations, the detector responses of interest were the axial-average ${ }^{16} \mathrm{~N}$ activation rates $(\mathrm{Bq} / \mathrm{atom})$ in each of the four inboard and two center flow tubes that contained nickel wires in the case of the ATRC Irradiations but which contain water in the case of the ATR. There are four additional detector flow tubes in the ATR as noted earlier but for clarity these were not considered in the analysis presented here since a comparison of results for the six-detector simplified model used here with results of a corresponding full 10-detector (11-equation) lobe power reconstruction model showed only minor differences.

Table 15 shows the reduced six-detector coefficient matrix, lobe powers and detector responses computed for Cycle 157D. The total power of the reactor was assumed to be $1 \mathrm{MW}$. The scaling factors used in this case for the seventh (total power) equation $\left(1.0 \times 10^{-21}\right)$ are not included in the table.

Table 15. Reduced coefficient matrix, computed lobe powers normalized to $1 \mathrm{MW}$ total, and computed ${ }^{16} \mathrm{O}(\mathrm{n}, \mathrm{p}){ }^{16} \mathrm{~N}$ interaction rates per atom for startup of Cycle 157D.

\begin{tabular}{|c|c|c|c|c|c|c|c|}
\hline Detector & NW Lobe & NE Lobe & SE Lobe & SW Lobe & Center Lobe & $\begin{array}{c}\text { Computed } \\
\text { Lobe Powers } \\
(\mathrm{kW})\end{array}$ & $\begin{array}{c}\text { Computed }{ }^{16} \mathrm{~N} \\
\text { Production Rate } \\
\left(\mathrm{Bq} /{ }^{16} \mathrm{O} \text { atom }\right)\end{array}$ \\
\hline $\mathrm{NW}$ & $4.784 \times 10^{-23}$ & & & & $8.372 \times 10^{-25}$ & $170.5(\mathrm{NW})$ & $8.329 \times 10^{-18}$ \\
\hline $\mathrm{NE}$ & & $4.853 \times 10^{-23}$ & & & $8.841 \times 10^{-25}$ & $155.8(\mathrm{NE})$ & $7.731 \times 10^{-18}$ \\
\hline $\mathrm{SE}$ & & & $5.205 \times 10^{-23}$ & & $8.539 \times 10^{-25}$ & $225.9(\mathrm{SE})$ & $1.193 \times 10^{-17}$ \\
\hline $\mathrm{SW}$ & & & & $5.300 \times 10^{-23}$ & $9.787 \times 10^{-25}$ & $233.8(\mathrm{SW})$ & $1.257 \times 10^{-17}$ \\
\hline $\mathrm{H} 3$ & $5.122 \times 10^{-24}$ & $1.131 \times 10^{-23}$ & $4.899 \times 10^{-24}$ & $1.842 \times 10^{-24}$ & $4.804 \times 10^{-23}$ & $214.0(\mathrm{C})$ & $1.451 \times 10^{-17}$ \\
\hline $\mathrm{H} 11$ & $5.211 \times 10^{-24}$ & $1.920 \times 10^{-24}$ & $4.803 \times 10^{-24}$ & $1.048 \times 10^{-23}$ & $5.217 \times 10^{-23}$ & & $1.594 \times 10^{-17}$ \\
\hline
\end{tabular}

As in the case of the ATRC Irradiations, filtering the Cycle 157D coefficient matrix to remove negligibly small entries did not significantly affect the reconstructed lobe powers. Table 16 shows the reduced coefficient matrix for Cycle 158A. Once again, filtering the coefficient matrix does not introduce any significant error. 
Table 16. Reduced coefficient matrix, computed lobe powers normalized to $1 \mathrm{MW}$ total, and computed ${ }^{16} \mathrm{O}(\mathrm{n}, \mathrm{p}){ }^{16} \mathrm{~N}$ interaction rates per atom for startup of Cycle 158A.

\begin{tabular}{|c|c|c|c|c|c|c|c|}
\hline Detector & NW Lobe & NE Lobe & SE Lobe & SW Lobe & Center Lobe & $\begin{array}{c}\text { Computed } \\
\text { Lobe Powers } \\
(\mathrm{kW})\end{array}$ & $\begin{array}{c}\text { Computed }{ }^{16} \mathrm{~N} \\
\text { Production Rate } \\
\left(\mathrm{Bq} /{ }^{16} \mathrm{O} \text { atom }\right)\end{array}$ \\
\hline $\mathrm{NW}$ & $4.974 \times 10^{-23}$ & & & & $8.581 \times 10^{-25}$ & $171.8(\mathrm{NW})$ & $8.725 \times 10^{-18}$ \\
\hline $\mathrm{NE}$ & & $3.958 \times 10^{-23}$ & & & $1.011 \times 10^{-24}$ & $183.5(\mathrm{NE})$ & $7.475 \times 10^{-18}$ \\
\hline $\mathrm{SE}$ & & & $5.263 \times 10^{-23}$ & & $8.917 \times 10^{-25}$ & $245.6(\mathrm{SE})$ & $1.308 \times 10^{-17}$ \\
\hline $\mathrm{SW}$ & & & & $5.307 \times 10^{-23}$ & $9.489 \times 10^{-25}$ & $190.4(\mathrm{SW})$ & $1.027 \times 10^{-17}$ \\
\hline $\mathrm{H} 3$ & $5.123 \times 10^{-24}$ & $1.095 \times 10^{-23}$ & $5.223 \times 10^{-24}$ & $1.762 \times 10^{-24}$ & $5.135 \times 10^{-23}$ & $208.8(\mathrm{C})$ & $1.528 \times 10^{-17}$ \\
\hline $\mathrm{H} 11$ & $5.378 \times 10^{-24}$ & $1.900 \times 10^{-24}$ & $5.195 \times 10^{-24}$ & $1.111 \times 10^{-23}$ & $4.988 \times 10^{-23}$ & & $1.512 \times 10^{-17}$ \\
\hline
\end{tabular}

Table 17 shows the ratios of the non-zero entries in the reduced coefficient matrix for Cycle 158A to the corresponding entries in the filtered matrix for Cycle 157D, along with the tally uncertainties for these ratios. It can be seen that the magnitudes coefficient shifts between the two ATR cycles are often smaller than was observed in the case of the coefficient shifts for ATRC Irradiation 15-2 relative to Irradiation 15-1. Furthermore, the coefficient for the NE detector response per unit NE lobe power decreased rather than increased in the case where the KJRR fuel was loaded (158A) relative to the case for the reference cycle (157D). These differences are attributable in part to the fact that the larger axial power peak that occurs in the NE lobe core fuel in Cycle 158A (KJRR present) relative to Cycle 157D (LSA cobalt irradiation hardware in NEFT) should not significantly affect the nitrogen activation rate in the northeast detector tube since the water being activated flows through the entire active length of the detector tube and the effect of the larger axial peak in Cycle 158A is largely averaged out, unlike the situation with the much more axially-localized nickel wire detectors used for the ATRC experiments.

Table 17. Ratios of filtered LPCIS system coefficients for Cycles 158A and 157D and their combined tally uncertainties.

\begin{tabular}{|c|c|c|c|c|c|}
\hline \multicolumn{6}{|c|}{ Coefficient Ratios (Cycle 158A/Cycle 157D) } \\
\hline Detector & NW Lobe & NE Lobe & SE Lobe & SW Lobe & Center Lobe \\
\hline NW & 1.040 & & & & 1.025 \\
\hline NE & & 0.816 & & & 1.144 \\
\hline SE & & & 1.011 & & 1.044 \\
\hline SW & & & & 1.001 & 0.970 \\
\hline $\mathrm{H} 3$ & 1.001 & 0.969 & 1.066 & 0.957 & 1.069 \\
\hline H11 & 1.032 & 0.990 & 1.082 & 1.060 & 0.956 \\
\hline \multicolumn{6}{|c|}{ Ratio Tally Uncertainties (\%) } \\
\hline Detector & NW Lobe & NE Lobe & SE Lobe & SW Lobe & Center Lobe \\
\hline NW & 1.60 & & & & 9.95 \\
\hline $\mathrm{NE}$ & & 1.70 & & & 9.06 \\
\hline SE & & & 1.33 & & 9.18 \\
\hline SW & & & & 1.40 & 8.84 \\
\hline $\mathrm{H} 3$ & 3.15 & 2.28 & 2.74 & 4.67 & 1.01 \\
\hline H11 & 3.09 & 5.31 & 2.74 & 2.08 & 1.00 \\
\hline
\end{tabular}




\section{[Type here]}

Furthermore, the coefficient shift differences may also be partially attributable to the fact that the change from the LSA cobalt irradiation hardware in the ATR NEFT for Cycle 157D to the KJRR experiment assembly for Cycle 158A has an opposite effect compared to the change in the ATRC from a water-filled NEFT to the KJRR element in the NEFT, in terms of changing the probability that neutrons produced in the core fuel elements in the lobe will reach the NEFT detector flow tube.

Table 18 shows the reconstructed lobe powers for Cycle 158A that are obtained using the filtered coefficient matrix for Cycle 157D and the computed detector responses for Cycle 158A and compares these to the case where the Cycle 158A lobe powers are reconstructed using the filtered coefficient matrix for Cycle 158A. The differences in lobe powers for all but the NE lobe are generally small, in the range of 5\% or less, and are largely within the propagated statistical uncertainties of the underlying coefficient matrix entries. In the case of the NE lobe however, the nominal $15 \%$ difference between the two reconstructed powers is statistically significant and indicates that unless the LPCIS system is recalibrated to reflect the coefficient shift between Cycles 157D and 158A the reconstructed power for the NE lobe may be indicated low by 13\%-17\%, with spread in the estimate being due to consideration of propagated tally uncertainties in the calculation.

Table 18. Reconstructed Lobe Powers (Watts) for Cycle 158A using reduced coefficient matrix for Cycle 157D and Cycle 158A using computed detector responses for Cycle 158A in both cases

\begin{tabular}{|c|c|c|c|}
\hline Lobe & $\begin{array}{l}\text { Reconstructed Power (kW) } \\
\text { Based On Cycle 157D } \\
\text { Coefficients }\end{array}$ & $\begin{array}{l}\text { Reconstructed Power (kW) } \\
\text { Based on Cycle 158A } \\
\text { Coefficients }\end{array}$ & $\begin{array}{l}\text { Ratio of Results with 157D } \\
\text { Coefficients to Results with } \\
\text { 158A Coefficients }\end{array}$ \\
\hline NW & 180.7 & 171.8 & 1.052 \\
\hline NE & 155.1 & 183.5 & 1.019 \\
\hline SE & 250.2 & 245.6 & 1.000 \\
\hline SW & 190.3 & 190.4 & 1.036 \\
\hline Center & 216.3 & 208.8 & \\
\hline
\end{tabular}

As an example of the needed calibration correction, Table 19 shows a set of trial multipliers for the 158A detector responses that will force the reconstructed powers for Cycle 158A that are obtained using the Cycle 157D coefficients with the Cycle 158A detector responses to be in reasonable agreement with the powers obtained when the Cycle 158A coefficient matrix is used for the same reconstruction. Table 20 then shows the result of the lobe powers that result from using these trial multipliers in the reconstruction equations.

Table 19. Trial multipliers for use with Cycle 157D coefficients combined with Cycle 158A detector responses.

\begin{tabular}{|c|l|l|l|}
\hline Detector & Multiplier & $\begin{array}{l}\text { Original } \\
\text { Response }\end{array}$ & $\begin{array}{l}\text { Modified } \\
\text { Response }\end{array}$ \\
\hline $\mathrm{NW}$ & 0.95 & $8.725 \times 10^{-18}$ & $8.288 \times 10^{-18}$ \\
\hline $\mathrm{NE}$ & 1.18 & $7.475 \times 10^{-18}$ & $8.820 \times 10^{-18}$ \\
\hline $\mathrm{SE}$ & 1.00 & $1.308 \times 10^{-17}$ & $1.308 \times 10^{-17}$ \\
\hline $\mathrm{SW}$ & 1.00 & $1.027 \times 10^{-17}$ & $1.027 \times 10^{-17}$ \\
\hline $\mathrm{H} 3$ & 1.00 & $1.528 \times 10^{-17}$ & $1.528 \times 10^{-17}$ \\
\hline $\mathrm{H} 11$ & 1.00 & $1.512 \times 10^{-17}$ & $1.512 \times 10^{-17}$ \\
\hline
\end{tabular}


Table 20. Cycle 158A Lobe powers computed using 157D Coefficients and modified 158A responses from Table 19.

\begin{tabular}{|c|c|c|c|}
\hline Lobe & $\begin{array}{c}\text { Reconstructed } \\
\text { Power }(\mathrm{kW})\end{array}$ & Target Power $(\mathrm{kW})$ & $\begin{array}{c}\text { Ratio of Reconstructed Power } \\
\text { to Target Power }\end{array}$ \\
\hline NW & 169.6 & 171.8 & 0.987 \\
\hline NE & 179.9 & 183.5 & 0.981 \\
\hline SE & 247.9 & 245.6 & 1.009 \\
\hline SW & 188.4 & 190.4 & 0.990 \\
\hline Center & 214.7 & 208.8 & 1.028 \\
\hline
\end{tabular}

Finally, it may be noted that at an intermediate-power hold point during the ascension to full cycle power during the actual startup of the ATR for Cycle 158A it was observed that the ATR NE quadrant power, as indicated by the LPCIS without applying any multipliers was in fact in the range of approximately $8 \%$ low relative to the corresponding calorimetric measurement for the same quadrant. This difference exceeded the maximum acceptable difference per the ATR operating procedures, and based on the observed trend it would have continued to grow as the power continued to rise. The observed difference was primarily attributed to a low indication of the power of the NW lobe, as expected based on the calculations and measurements presented here. When the recommended (Table 19) multipliers were then applied to the LPCIS reconstruction during the remainder of the startup, the resulting indicated quadrant powers from the LPCIS were much more consistent with the calorimetrically-measured powers for all four quadrants, satisfying the startup criteria, and full power was successfully achieved

\subsection{DISCUSSION}

The computational and experimental results reported here have demonstrated that the neutronic behavior of the KJRR fuel element in the ATRC is well-understood, both in terms of its general effects on ATRC core reactivity and fission power distributions, its effects on the calibration of the core lobe power measurement (LPCIS) system, as well as in terms of its own internal fission rate distribution and total fission power per unit ATRC core power. Taken as a whole, these results serve to extend the ATR physics validation envelope, enabling an entire new class of irradiation experiments in the ATR.

The measured axial and transverse fission power distributions within the KJRR fuel element were consistent with the corresponding computations as well as with expectations based on general physical considerations. The total measured fission power generated in the KJRR fuel element in the ATRC was approximately 7\% less than the computed value, on a constant-lobe-power basis. This difference is comparable to the combined statistical uncertainties of the calculation and the measurement at the $1 \sigma$ level and it is not of practical significance in terms of reactor safety considerations.

Based on MCNP5 computations and corresponding validation measurements performed in the ATRC, the KJRR fuel element was shown to have the potential to distort the LPCIS coefficient matrix to a degree sufficient to warrant concern about the accuracy of the LPCIS system unless it is recalibrated to reflect the presence of the KJRR fuel element in the Northeast Flux Trap. In the case of the planned initial irradiation of the KJRR fuel element during Cycle 158A in the ATR, calculations based on procedures developed, verified, and validated for the simulated LPCIS system in the ATRC suggest that the magnitudes of estimated shifts in the LPCIS coefficient matrix entries for ATR Cycle 158A relative to the assumed baseline cycle (157D) are somewhat smaller than in the ATRC bounding test cases but they are statistically significant and may range up to approximately $18 \%$ for the four inboard and two ${ }^{16} \mathrm{~N}$ detector positions of primary interest. These coefficient shifts may, in turn, cause the LPCIS system as programmed for Cycle 157D to produce an indication for the power of the NE lobe that is in the range of 13\%-17\% low in Cycle 158A relative to the independent calorimetric power measurement system.

Finally it is important to re-emphasize that the uncertainties quoted in this work for computed quantities are largely based on propagated MCNP5 tally uncertainties only. Other sources of uncertainty exist, especially those traceable to the estimated uncertainties of the burnup of each of the previously-irradiated ATR core fuel elements installed in 


\section{[Type here]}

the NE lobe for both the reference cycle (157D) and the target cycle (158A). These particular sources of uncertainty are essentially unquantifiable in a rigorous sense due to the current lack of recent, well-qualified, direct exposure validation data for irradiated ATR fuel elements that are in the inventory. However, empirical estimates, supported by indirect experimental evidence and informed engineering judgement, suggest that an additional uncertainty that could range up to about $5 \%$ may be associated with core fuel burnup-related sources.

\section{ACKNOWLEDGEMENTS}

This work was financially supported by the Korean Atomic Energy Research Institute. The authors also wish to gratefully acknowledge the essential technical contributions of INL Staff Members A. A. Beasley, D. J. Schoonen, C. D. Jackson, T. C. Unruh, M. Morrison, M.R. Holtz, R.A. Jordan, J. Shaw, D. T. Miller, L. D. Smith, G. K. Taylor, and S. G. Louk.

\section{REFERENCES}

1. Studsvik Scandpower, HELIOS Methods Version 1.10 (2008).

2. M.D. DeHart, "Advancements in Generalized-Geometry Discrete Ordinates Transport for Lattice Physics Calculations", Proc. of PHYSOR-2006, American Nuclear Society Topical Meeting on Reactor Physics: Advances in Nuclear Analysis and Simulation, Vancouver, British Columbia, Canada, September 10-14, 2006.

3. J.M. McGhee, T.A. Wareing, D.J. Barnett, ATTILA Version 5: User Manual, Transpire Inc., Gig Harbour WA, USA (2006).

4. D.F. Hollenbach, L.M. Petrie, N.F. Landers, KENO-VI: A General Quadratic Version of the KENO Program, ORNL/TM-13011, Oak Ridge National Laboratory, 1996.

5. T. Goorley, J. Bull, F. Brown, et al. Release of MCNP5_RSICC_1.30, LA-UR-04-4519, Los Alamos National Laboratory (2004).

6. B.L. Broadhead, B.T. Rearden, C.M. Hopper, J.J. Wagschal, C.V. Parks, "Sensitivity- and Uncertainty-Based Criticality Safety Validation Techniques,” Nucl. Sci. Eng. 146, 340-366 (2004).

7. T.M. Sutton, et al., The MC21 Monte Carlo Transport Code, Knolls Atomic Power Laboratory and Bettis Laboratory, LM-06K144 (2007).

8. J. Leppänen, Serpent Progress Report 2011, VTT-R-05444-12, Technical Research Centre of Finland (2012).

9. D. W. Nigg, J.W. Nielsen, B.M. Chase, R.K. Murray, K.A. Steuhm, T. Unruh, "Improved Computational Neutronics Methods and Validation Protocols for the Advanced Test Reactor", Proceedings of American Nuclear Society Topical Meeting on Advances in Reactor Physics - PHYSOR 2012, Knoxville, Tennessee, USA, April 1520, 2012.

10. D.W. Nigg, J.W. Nielsen, G.K. Taylor, "Validation Protocols to Support the Neutronics Modeling, Simulation, and V\&V Upgrade for the Advanced Test Reactor", Trans. ANS, 106:890-893 (2012)

11. D.W. Nigg and K.A. Steuhm (Editors), Advanced Test Reactor Core Modeling Update Project Annual Report for Fiscal Year 2014, INL/EXT-14-33319, September 2014.

12. J.W. Nielsen, D.W. Nigg, A.W. LaPorta, A Fission Matrix Based Validation Protocol for Computed Power Distributions in the Advanced Test Reactor, Nucl. Eng. Des., 295:615-624 (2015).

13. J.W. Nielsen, D.W. Nigg, and J.S. Kim, "MCNP5 Validation with a High-Reactivity Experiment in the Advanced Test Reactor Critical Facility", Trans. ANS 113:1049-1051 (2015).

14. JW Rogers, "Fission Rate Measurements in Fuel Plate Type Assembly Reactor Cores", IAEA document available at (https://inis.iaea.org/search/search.aspx?orig_q=RN:23023006).

15. J.L. Durney and N.C. Kauffman, "Calculating Reactor Power from Activation Techniques as Applied to an Unusual Fuel Geometry, IN-1047 (1967).

16. J.W. Nielsen, D.W. Nigg, N. Woolstenhulme, A.Beasley, "An Analysis Method for Experiment Power in the 
[Type here]

ATR", Trans. ANS 115:1347-1350 (2016).

17. Meyer, S.L., Data Analysis for Scientists and Engineers, John Wiley and Sons, 1975.

18. P.J. Griffin and R. Paviotti-Corcuera, "Summary Report of the Final Technical Meeting on "International Reactor Dosimetry File: IRDF-2002”, International Atomic Energy Agency, INDC(NDS)-448 (2003) 